Alma Mater Studiorum - Università di Bologna DEPARTMENT OF ECONOMICS

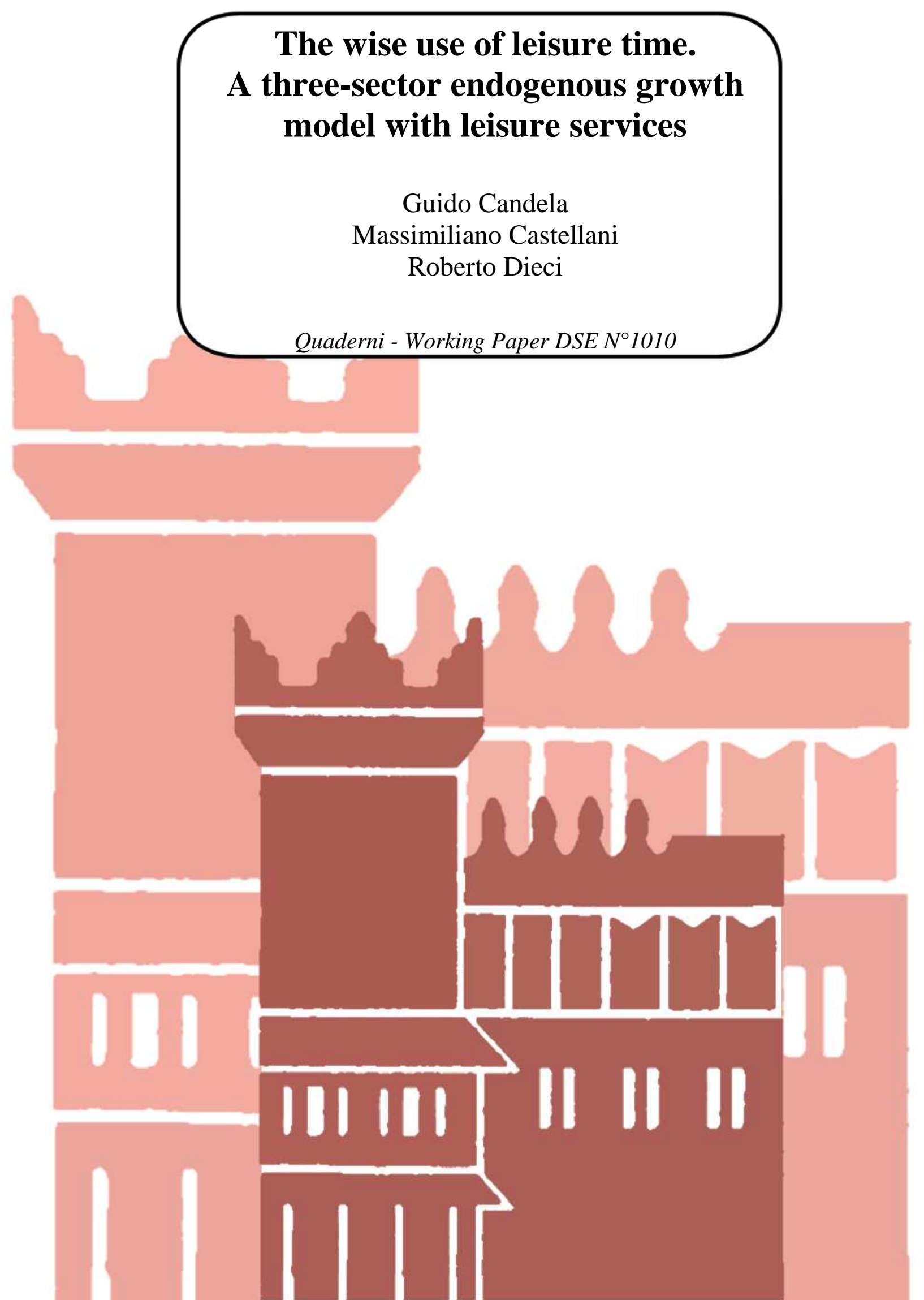




\title{
The wise use of leisure time. A three-sector endogenous growth model with leisure services
}

\author{
G. Candela*, M. Castellani**, R. Dieci*** \\ University of Bologna
}

June 1,2015

\begin{abstract}
In this paper, starting from the two-sector Uzawa-Lucas model, we study a three-sector endogenous growth model with leisure services. By extending the endogenous growth model with leisure developed by Ladrón-de Guevara et al. [1999], our model generalizes the standard time allocation problem, in that it explicitly accounts for the way total time is allocated between work, education, purely free time and time spent on leisure services, where the latter represents therefore an additional time-consuming activity. Accordingly, service consumption is tied to a production sector for leisure services in our model. We fully characterize Balanced Growth Path (BGP) equilibria in terms of time allocation and growth, and show that multiple BGPs are possible. Since service production plays an important role in economic development, we carry out a comparative analysis of the dynamic performance of different economic systems - Post-Industrial Economy vs. Service Economy - along a BGP.
\end{abstract}

\section{JEL Classification: O14, O41, D90} Keywords: Leisure, Service Economy, Multisector Growth Model

Acknowledgement We would like to thank Corrado benassi for helpful comments on a previous version of the paper. The usual disclaimer applies.

* Guido Candela

Dept. of Economics, University of Bologna

e-mail: guido.candela@unibo.it

** Massimiliano Castellani (corresponding author)

Dept. of Economics, University of Bologna, Italy

Tel.: 00390512098020 Fax: 0039051221968

Piazza Scaravilli 240125 Bologna, Italy

e-mail: m.castellani@unibo.it

*** Roberto Dieci

Dept. of Mathematics, University of Bologna, Italy

e-mail: roberto.dieci@unibo.it

\section{Introduction}

One of the main contributions of optimal growth theory is having pointed out that economic systems are characterized not only by social and industrial relations, but also by the way time is allocated between different activities by agents. Within endogenous growth theory, the Uzawa-Lucas model deals with the problem of optimal time allocation for economic development. In particular, Lucas [1988] 
assumes that agents devote a part of their time to increase their human capital (educational time). Lucas' assumption on educational time implies that human capital becomes an endogenous dynamic variable and an engine of economic development. Starting from Uzawa [1965] hypothesis, Lucas [1988] develops a two-sector growth model, by adding to the physical production sector another sector in which human capital is produced. Following this seminal model, several authors (e.g. Mulligan and Sala-i Martin [1993], Benhabib and Perli [1994], Bond et al. [1996], and references therein) have analyzed the steady state dynamics (existence, uniqueness, and saddle-path stability of the balanced growth equilibrium) and transitional dynamics of two-sector endogenous growth models with human and physical capital.

More recently, Ladrón-de Guevara et al. [1999] consider two extensions of the Uzawa-Lucas model. In the first one, physical capital is included as an input of the educational sector. In the second, leisure choices play a role in agents' welfare. Unlike the Uzawa-Lucas framework, the model with leisure can have a multiplicity of steady states with different rates of growth. On the basis of this model a strand of literature has explored several hypotheses on time allocation and our paper fits into this literature. In particular Ortigueira [2000] assumes that the representative consumer derives utility from consumption and qualified leisure and proves the existence of a unique globally stable balanced growth path equilibrium. By assuming that the utility function of the representative agent is not additively separable between consumption and leisure time, Mino [2002] finds that indeterminacy may occur. Psarianos [2007] introduces leisure as a choice variable in the utility function and argues that this hypothesis reduces the growth rate of the economy, even though agents are willing to accept a lower rate of growth of income in exchange for leisure time. Azariadis et al. [2013], by assuming an utility function that is separable in consumption and leisure or leisure externalities, indicate that a higher preference for leisure or leisure externality implies less growth but also lower education attainment.

In the Uzawa-Lucas framework total available time is allocated between working time (the time individuals spend at work) and leisure time (the time individuals devote to themselves). However, such optimal growth models do not explicitly consider the way leisure time is used by agents. This indeed remains an open issue, as leisure time is not completely absorbed by pure leisure (free time) or educational time in reality, but also by the consumption of services, which generally represents a time-consuming activity. ${ }^{1}$ In other words, although leisure time affects agents' utility in this literature, it is implicitly treated as 'wasted' time, in that it does not feed back to the economy directly, nor can it support physical and human capital accumulation. In contrast, both production and accumulation of physical and human capital are directly affected by the way leisure time is allocated between pure leisure and the time-consuming services.

If agents' utility function has an additional argument - the time share absorbed by consumption of services - and, symmetrically, a production sector for leisure services is to be introduced, this has two consequences in a standard optimal growth framework. First, leisure time needs to be optimally reallocated between pure leisure and time-consuming services. Second, since leisure may now sustain aggregate demand and the production of leisure services, it turns out to play an important role in economic development. However, as mentioned above, this crucial aspect has been neglected in endogenous growth theory so far.

To fill this gap, in this paper we propose a novel three-sector growth model, describing the joint dynamics of the industrial sector and the service sector - both employing physical and human capital as input factors - and that of the education sector. ${ }^{2}$ Our assumption generalizes the time allocation

\footnotetext{
${ }^{1}$ Following Becker [1965], we mean time-consuming services those leisure services, such as art, music, entertainment, wellness, tourism, etc., whose consumption require a portion of an agent's time (leisure). Some time-consuming services do not have good market substitutes, since they are self-produced by consumers. In this paper we consider only marketable services. For an alternative approach when the 'consumption takes time' see Steedman [2003].

${ }^{2}$ Schultz [1961] introduced the idea of the investment in human capital and Becker [1965] developed the first general
} 
problem and, as a consequence, the extensions of the Uzawa-Lucas model dealing with leisure can be regarded as particular cases of our general model. Despite this generalization, our model remains partial in that we do not endogenize the optimal factor mix to be used in the industrial and service production. $^{3}$

Starting from the Uzawa-Lucas model and adding a third sector in which time-consuming services are produced, we study the Balanced Growth Path (BGP) equilibrium of an economic system (which we will call Service Economy) in which leisure time is allocated between free time and service consumption time, and in which a social planner makes all intertemporal choices. The discussion and interpretation of our results is largely based on a comparison with Ladrón-de Guevara et al. [1999]. We identify in this model a relevant particular case of our model, corresponding to an economic system without the service sector (which we will call Post-Industrial Economy) and carry out a comparison of the BGP dynamics of the two economies. We focus on a general characterization of BGP equilibria in terms of time-allocation, growth rates and welfare, but this paper is not specifically focused on the question of transition dynamics to long-run equilibria and that of their (saddle-path) stability. Although the latter do represent important issues in modern growth theory, our BGP analysis alone provides an interesting picture of the impact of services consumption and production on endogenous growth.

Since the dynamic efficiency problem concerns both productive and allocative efficiency conditions of the short and long run equilibrium, a comparative analysis of the dynamic performance of different economic systems along BGP equilibria should rely upon two efficiency criteria, in our view: a 'social welfare' criterion (maximized utility), measuring allocative efficiency, and a 'wealth' criterion (the ouput growth rate along a BGP), which captures productive efficiency. ${ }^{4}$

The remainder of the paper is organized as follows. In Section 2 we set up our endogenous growth model and discuss the social planner program in an economic system with and without leisure services. We also illustrate how the standard two-sector growth model with leisure is nested in a three-sector growth model with leisure services. Section 3 deals with the general properties of the BGP equilibria of our model, also in comparison with the BGP equilibria of the two-sector model. By assuming CES utility and Cobb-Douglas production technology for both production sectors, Section 4 illustrates how the BGPs of our model can be determined in general and provides evidence of multiple equilibria for reasonable parameter values. Section 5 draws some conclusions and points out some directions for future research. Section 6 collects a number of mathematical appendices about the main results of the paper.

\section{Theoretical framework}

The way individuals allocate time spent away from work is crucial to understand the main economic activities of modern economies. We define as Post-Industrial Economy (PE) the economic system

treatment of allocation of time in all non-work activities. More recently, Rogerson [2007] and El-hadj [2009] have developed a version of the neoclassical growth model with three sectors (agriculture, manufacturing and services) to study structural transformation, but none of them pays attention to the services consumption time.

${ }^{3}$ Echevarria [1997], Ngai and Pissarides [2007], Rogerson [2007] and El-hadj [2009], assume exogenous capital shares in all sectors. Ngai and Pissarides [2007] also analyze the structural change in a multi-sector growth model, when capital shares are different across sectors.

${ }^{4}$ Comparing levels of output per capita is another wealth criterion, but it requires additional information on relative prices as compared with the criterion based on growth rate of output per capita. However, in the long run and starting from any time, the system with the highest growth rate of output per capita will become the system with the highest level of output per capita. To evaluate the dynamic efficiency, Wong and Yip [1999] analyze the (gross) growth rate of welfare of individuals (over two consecutive generations) as a measure of dynamic welfare and Chu [2012] highlights the importance of a dynamic welfare effect of international transfers through economic growth. 
in which total available time is allocated between work, leisure and education. We define as Service Economy (SE) the economic system in which part of leisure time is absorbed by the consumption of services and only the rest is purely free time. Thus, in the SE total available time is allocated between work, leisure and educational time, and in turn leisure is allocated between free time and service consumption time.

The PE is an economic system that involves two economic activities: industrial and educational activity. This economic system is characterized by the presence of a single market, matching supply and demand for the industrial good, whereas the additional educational activity can only be accumulated to increase human capital. In contrast, in the SE agents' allocation of leisure time generates market demand for leisure services, whereas market supply for services requires a production activity that employs capital and labor. Therefore, a stylized description of this economic system characterized by three activities (industrial, services and educational activity), requires modeling two markets: one for the industrial good and another for leisure services. ${ }^{5}$

In this Section we set up a growth model for the SE, which offers a wider range of options for time allocation than the $\mathrm{PE}$ does. Accordingly, it is characterized by an increased number of markets and activities. Put differently, the SE encompasses PE as a special case, once suitable restrictive assumptions are introduced. ${ }^{6}$ A final comment is in order about the level of generality of our setup. In a sense, introducing demand and supply of time-consuming services is not just adding one more sector. The resulting enlarged model of the SE displays a complete 'taxonomy' of sectors and activities according to whether or not equilibrium condition (market clearing) at each point in time implies intertemporal stock adjustments: i) equilibrium in the industrial sector entails an immediate effect, via the consumption flow at the time of production, as well as the intertemporal accumulation of the stock of physical capital; ii) the educational activity involves a purely intertemporal effect, since the stock of knowledge can only be accumulated to increase human capital; iii) finally, equilibrium in the service sector can be attained solely through consumption at the same time of production, since leisure services cannot be stored over time. For this reason, as shown in Section 2.2, the instantaneous equilibrium conditions for the industrial good and for the educational activity are formulated through differential equations, as usual, whereas leisure services are characterized by a 'static' equilibrium condition, such that this equilibrium at any time is self-contained and may be determined by current parameters only.

Preliminary to our BGP analysis in Section 3, in this Section we set up a three-sector model with leisure services by spelling out the model assumptions (Section 2.1), by defining the equilibrium conditions and laws of motion for each sector (Section 2.2) and by deriving the intertemporal program for the SE (Section 2.3). For the sake of completeness, in Section 2.3 we discuss and interpret the SE as a generalization of the PE.

\section{$2.1 \quad$ Model setup}

We introduce our notation through a brief discussion of the effect of enlarging the choice set for agents' time allocation. If total time available to the individual is normalized to one, the starting point is the

\footnotetext{
${ }^{5}$ Since both physical and human capital should be allocated to several activities, the full employment of productive factors needs a complete system of relative prices. Furthermore, income in the PE is the amount of industrial goods, while in SE (where production is diversified into industrial goods and leisure services) total income corresponds to the total value of these products. In any case, an aggregate measure always requires a price system.

${ }^{6}$ Furthermore, through further restrictions on time allocation, we can obtain the Ramsey-Cass-Koopmans growth model [Ramsey, 1928, Cass, 1965, Koopmans, 1963] for an economic system in which no time is devoted to education, and the Solow growth model [Solow, 1956] for an economic system in which there is no free time.
} 
case where total time is devoted to working activities $(u)$, namely:

$$
1=u
$$

whereas allocation of available time between working time and free time ( $\mu$, the fraction of time individuals devote to themselves), is formalized as:

$$
1=u+\mu .
$$

Introduction of educational time $(\lambda)$, devoted to human capital formation, results in the following time allocation structure:

$$
1=u+\mu+\lambda
$$

However, in our model free time $\mu$ is not just identified with pure leisure time $(l)$, but it also includes time needed for consumption of services $(s)$ :

$$
1=u+\lambda+s+l \text {. }
$$

Put differently, we acknowledge that the existence of a production sector for services and of a market that matches supply and demand of leisure services necessarily requires individuals to spend a portion of their time to consume such services.

As will be clear in sequel, it is formally convenient to determine the amount of education time in the SE residually:

$$
\lambda=1-u-l-s .
$$

For strictly positive $u, \lambda, l, s$, we may regard equation (5) as a characterization of the SE in terms of time allocation. Obviously, equation (5) generalizes previous allocation models for proper restrictions on the variables.

In the following, we adopt a continuous time setup and we denote by $\dot{x}=\frac{d x}{d t}$ the time derivative of variable $x=x(t)$, as usual. Furthermore, we omit the explicit indication of time when it is unnecessary.

Population, $N(t)$, is an exogenous variable corresponding to the available number of workers (full employment hypothesis) and growing at a constant exogenous rate, $n$. In addition, the initial size of the population is normalized to one $(N(0)=1)$. The population dynamics is given by the following standard equation:

$$
N(t)=\exp (n t)
$$

The total stock of human capital available at time $t(H(t))$ is given by the number of workers at time $t(N(t))$ multiplied by the average level of human capital of each worker $(h \geq 1)$ :

$$
H(t)=h(t) N(t)
$$

The time derivative of human capital thus reads: $\dot{H}=\dot{h} N+h \dot{N}$. At any time $t$, human capital is fully employed in the production of industrial goods and of leisure services.

The total stock of physical capital is denoted by $K(t)$ and its time derivative defines (net) investments $\dot{K}=I(t)$. $H(t)$ and $K(t)$ are endogenous variables of the model.

The SE comprises three sectors: industrial, educational, and leisure service sectors. A fraction $\gamma$ of available physical capital and a fraction $\delta$ of available human capital are used to produce the industrial good, $Y(t)$. The production function of the industrial good is given by: ${ }^{7}$

\footnotetext{
${ }^{7}$ We disregard the exogenous growth due to technological progress. However, the effect of technological progress on growth can be introduced in our model, via a non-autonomous version of equation (8).
} 


$$
Y(t)=f(\gamma K, \delta u H), \quad f(0, .)=0, f(., 0)=0 .
$$

As usual, this production function is increasing with respect to physical and human capital and twice differentiable. By assuming additionally that function $f$ is homogeneous of degree one, equation (8) can be rewritten in per capita terms using $y:=Y / N, k:=K / N$ and $h:=H / N$ :

$$
y=f(\gamma k, \delta u h) .
$$

The process of human capital accumulation has been described in the literature in several ways. On the one hand, Lucas [1988] assumes that the growth of human capital depends directly on educational time. On the other hand, Bond et al. [1996] assume a process that employs both physical and human capital. In our model we stick to the Lucas hypothesis by assuming, as a first approximation, that production of new human capital $X(t)=\dot{H}$ depends on the fraction of time spent by employees for their education given the average level of human capital:

$$
X(t)=q(\lambda h N), \quad q(0)=0,
$$

where $q$ is a strictly increasing function of its argument, as well as homogeneous of degree 1. By dividing $X($.$) by N$, equation (10) can thus be rewritten in per capita terms:

$$
x(t)=X / N=q(\lambda h)=\psi \lambda h,
$$

where $\psi>0$ is the marginal productivity of time spent on education.

Under full employment conditions, production of leisure services, $Z(t)$, absorbs the amount of physical and human capital which is not used in industrial production. Then, the production function for services is given by:

$$
Z(t)=g((1-\gamma) K,(1-\delta) u H), \quad g(0, .)=0, g(., 0)=0,
$$

where $g$ is also increasing with respect to physical and human capital and twice differentiable. Again, by assuming that function $g$ is homogeneous of degree one, we may rewrite equation (12) in per capita terms:

$$
z(t)=Z / N=g((1-\gamma) k,(1-\delta) u h) .
$$

In our model we take $\gamma$ and $\delta$ as exogenous. A more general model should endogenize these quantities and treat them as further control variables $\gamma(t)$ and $\delta(t)$ in the social planner's allocation problem. We do not care about this issue here for three reasons. First, we investigate the effects of expanding the choice set in terms of time allocation alternatives, and of introducing an additional economic sector accordingly: treating the allocation of input factors across sectors as endogenous would largely increase the dimension of the model, and drive our attention away from our main objective. Second, our paper entirely focuses on the determination and characterization of the BGP of the economy, and the assumption of constant factor allocations across sectors is consistent with the dynamics in a BGP equilibrium. Third, our main findings about growth rates and time allocation in a BGP are rather general and do not depend on sectoral allocation parameters, $(\gamma, \delta){ }^{8}$

\footnotetext{
${ }^{8}$ As well as in Mankiw et al. [1992], the allocation of the available resources between physical and human capital investment is exogenously given. A recent stream of literature on economic development explains the cross-country differences in the growth rates of GDP as the result of differences in the sectoral composition of GDP [Echevarria, 1997, Laitner, 2000]). More recently, Chanda and Dalgaard [2008], Córdoba and Ripoll [2009], Alonso-Carrera and Raurich [2010] show that changes in the sectoral composition may contribute both to the output and productivity growth, without any changes in total factor productivity (technological changes).
} 


\subsection{Equilibrium conditions, consumption and asset accumulation}

For each of the three goods/sectors of the SE, a suitable equilibrium condition needs to be imposed to an optimal growth path.

Equilibrium for the industrial good implies that (net) investment in physical capital, $I(t)=\dot{K}(t)$, is equal to (net) savings, $S(t)=Y(t)-C(t)-\sigma K(t)$ :

$$
\dot{K}=Y-C-\sigma K,
$$

where $C$ is consumption and $\sigma>0$ is the instantaneous constant rate of physical capital depreciation. As usual, dividing equation (14) by $N$ allows to rewrite the equilibrium condition in per capita terms:

$$
\frac{\dot{K}}{N}=y-c-\sigma k
$$

where $c:=C / N$ is per capita consumption, and $\dot{K} / N$ is per capita investment in physical capital.

Equation (15) is the usual form of physical capital accumulation. By definition of $k$ we obtain $\dot{k}=(\dot{K} N-K \dot{N}) / N^{2}$ or $\dot{K} / N=\dot{k}+n k$, and by substitution in equation (15) we can rewrite it as a law of motion of per capita variables:

$$
\dot{k}=y-c-(\sigma+n) k .
$$

We assume that knowledge tends to becomes obsolete over time, and denote by $\theta \geq 0$ the instantaneous and constant rate of human capital depreciation. As time devoted to study increases human capital directly, equilibrium for the educational activity is obtained from equation (10):

$$
\dot{H}=X-\theta H=\psi \lambda h N-\theta H .
$$

Again, equilibrium equation (17) for the educational activity can be rewritten in per capita terms:

$$
\frac{\dot{H}}{N}=\psi \lambda h-\theta h,
$$

where $\dot{H} / N$ is per capita investment in human capital. From the definition of $h$ we obtain $\dot{h}=$ $(\dot{H} N-H \dot{N}) / N^{2}$ or $\dot{H} / N=\dot{h}+n h$, and therefore dynamic equation (18) can be reformulated in per capita variables too, in a way similar to (16):

$$
\dot{h}=\psi \lambda h-(\theta+n) h .
$$

If human capital does not suffer a 'dilution effect' and knowledge is transmitted to new generations genetically, we can neglect term $n h$ in equation (19). By Using condition (5), we can rewrite (19) as:

$$
\dot{h}=\psi(1-u-l-s) h-\theta h .
$$

As discussed above, consumption of leisure services requires time. In addition, service consumption is proportional to the level of human capital. Therefore we obtain:

$$
Z=\operatorname{sh} N
$$

or again in per capita terms:

$$
z:=\frac{Z}{N}=s h
$$


Due to their nature of pure services, leisure services cannot be stored and therefore their production necessarily matches consumption at each point in time. A market clearing condition for this sector is simply obtained by combining equations (13) and (22):

$$
g((1-\gamma) k,(1-\delta) u h)-s h=0
$$

\subsection{Social welfare and intertemporal programs}

The economy is populated by an infinitely-lived (dynasty of the) representative agent. The representative agent consumes goods and services and allocates her time between work, education, leisure time and time-consuming services. If the representative agent is not affected by any 'efficiency illusion' (i.e. efficiency has no effect on the utility function), her utility depends on the actual time share devoted to service consumption, so that the utility function of the representative agent is $U(c, l, s)$, where $s=z / h$ represents service consumption per unit of human capital. Function $U($.$) is continuous, twice$ differentiable, strictly concave and increasing in $c, l, s$. The social planner wants to maximize social welfare, expressed by the following functional:

$$
\int_{0}^{\infty} N U(c, l, s) \exp (-\rho t) d t
$$

where $\rho$ is the intertemporal discount rate (time preference rate).

Substitution of equation (6) into equation (24) leads to the standard formulation:

$$
\left.\int_{0}^{\infty} U(c, l, s) \exp (n-\rho) t\right) d t
$$

which requires the usual consistency condition $\rho>n$.

By taking into account constraints (16), (20) and (23), as well as time constraint (5), and for given initial values of state variables, the social planner's intertemporal maximization problem in the SE can be recast as follows:

$$
\begin{aligned}
& \left.\max \int_{0}^{\infty} U(c, l, s) \exp (n-\rho) t\right) d t \\
\text { s.t. } \dot{k} & =f(\gamma k, \delta u h)-c-(\sigma+n) k \\
\dot{h} & =\psi(1-u-l-s) h-\theta h \\
0 & =g((1-\gamma) k,(1-\delta) u h)-h s \\
k(0) & =k_{0}, h(0)=h_{0} \\
u, l, s & \geq 0,0 \leq l+u+s \leq 1 .
\end{aligned}
$$

Optimal control program (26) has four control variables $(c, u, l, s)$, two state variables $(k, h)$ and one static constraint for the service sector, besides the standard feasibility constraints on the controls. Note that $\lambda=1-u-l-s$. The solution involves two co-state variables $\left(\pi_{1}, \pi_{2}\right)$ associated to the laws of motion of $k$ and $h$, respectively, and an additional multiplier $\pi_{3}$ associated to the static constraint. Co-state variables and the multiplier can be interpreted as shadow prices in the social valuation of goods and services. As discussed above, the values $\gamma$ and $\delta$ are assumed as parameters. Program (26) for the SE includes the social planner's dynamic optimization problem for PE as particular case, as briefly discussed below.

By definition, in the $\mathrm{PE}$ the representative agent devotes no time to the consumption of leisure services and all free time is spent as pure leisure time. From imposing $s=0$ ( or $\mu=l$ ) it follows 
that $z(t) \equiv 0$ in equation (22). Consistently, equation (13) requires $\gamma=1$ and $\delta=1$, which reduces equation (23) to an identity. Therefore, the optimal control problem for the PE is expressed as:

$$
\begin{aligned}
&\left.\max \int_{0}^{\infty} U(c, l) \exp (n-\rho) t\right) d t \\
& \text { s.t. } \dot{k}=f(k, u h)-c-(\sigma+n) k \\
& \dot{h}=\psi(1-u-l) h-\theta h \\
& k(0)=k_{0}, h(0)=h_{0} \\
& u, l \geq 0,0 \leq u+l \leq 1,
\end{aligned}
$$

which has three control variables, $(c, u, l)$, and two state variables $(k, h)$ and where $\lambda=1-u-l$. The solution of program (27) involves two co-state variables $\left(\pi_{1}, \pi_{2}\right)$.

\section{Balanced Growth dynamics}

The goal of this Section is wide-ranging. First, after setting up the necessary optimality conditions, we derive the BGP conditions for the SE (Section 3.1). Second, we compare different BGP equilibria in the SE. Our results apply to both the case of multiple BGPs in the phase space and the case of a unique BGP under different parameter constellations. This will highlight the impact of time allocation on both social welfare and growth of the economy (Section 3.2). Third, we derive the BGP conditions for the economy modeled and investigated in Ladrón-de Guevara et al. [1999], which can be classified as PE in our general framework (Section 3.3). Fourth, we perform a comparative analysis between the BGP dynamics of the two economies (Section 3.4).

Denote by $f_{K}, f_{L}, g_{K}, g_{L}$ the partial derivatives of $f$ and $g$ with respect to physical and human capital, respectively. As shown in Appendix 6.1, an interior optimal solution to program (26) needs to satisfy the following set of first-order conditions:

$$
\begin{gathered}
U_{c}(c, l, s)=\pi_{1} \\
U_{l}(c, l, s)=\pi_{2} h \psi \\
\delta \pi_{1} f_{L}(\gamma k, \delta u h)+(1-\delta) \pi_{3} g_{L}((1-\gamma) k,(1-\delta) u h)=\pi_{2} \psi \\
U_{s}(c, l, s)=\pi_{2} h \psi+\pi_{3} h \\
\frac{\dot{\pi}_{1}}{\pi_{1}}=\rho+\sigma-\gamma f_{K}(\gamma k, \delta u h)-R(u, k, h) \frac{1}{h}\left[\frac{U_{l}(c, l, s)}{U_{c}(c, l, s)}-h \delta f_{L}(\gamma k, \delta u h)\right] \\
\frac{\dot{\pi}_{2}}{\pi_{2}}=(\rho-n)+\theta-\psi(1-l)+\psi s \frac{U_{s}(c, l, s)}{U_{l}(c, l, s)},
\end{gathered}
$$

where:

$$
R(u, k, h)=\frac{(1-\gamma) g_{K}((1-\gamma) k,(1-\delta) u h)}{(1-\delta) g_{L}((1-\gamma) k,(1-\delta) u h)} .
$$

Conditions (28)-(31) are the standard stationarity conditions of the Hamiltonian of (26) with respect to the control variables $c, l, u, s$. Note that the laws of motion of the costate variables $\pi_{1}$ and $\pi_{2}$ are conveniently rewritten without the explicit appearance of $\pi_{1}, \pi_{2}$ and $\pi_{3}$ at the right-hand sides of (32) and (33), as shown in Appendix 6.1. In addition to (28)-(33), the following constraints from program (26) must be fulfilled:

$$
\dot{k}=f(\gamma k, \delta u h)-c-(\sigma+n) k
$$




$$
\begin{gathered}
\dot{h}=\psi(1-l-s-u) h-\theta h \\
g[(1-\gamma) k,(1-\delta) u h]=s h .
\end{gathered}
$$

In Appendix 6.2 we specialize conditions (28)-(33) to the standard case of CES utility, consistent with, e.g. Ladrón-de Guevara et al. [1999]. More precisely, we will assume a utility function of the form:

$$
U(c, l, s)=\frac{1}{1-\varepsilon}\left(c^{a_{1}} l^{a_{2}} s^{a_{3}}\right)^{1-\varepsilon},
$$

where $\varepsilon>0, \varepsilon \neq 1,0<a_{i} \leq 1(i=1,2,3), \sum_{i=1}^{3} a_{i}=1$ and, as usual, we will extend (38) to include the case $\varepsilon=1$, in which case function $U$ reduces to log-utility:

$$
\widetilde{U}(c, l, s)=a_{1} \ln c+a_{2} \ln l+a_{3} \ln s .
$$

\subsection{BGP conditions for the SE}

We are interested in characterizing the BGP solutions for the SE, namely, the optimal solutions $(c(t), l(t), u(t), s(t), k(t), h(t))$ to program (26) for some initial condition $k(0)=k_{0}, h(0)=h_{0}$, such that $c, k$ and $h$ grow at constant rates, $l, u$ and $s$ remain constant and the output/capital ratios $y / k$ and $z / k$ are constant too. It is straightforward to show that $c, k$ and $h$ need therefore to grow at the same constant rate, say $\nu$, along a BGP. As shown in Appendix 6.3, a BGP is expressed as a 6-tuple $\left((c / k)^{*},(h / k)^{*}, l^{*}, u^{*}, s^{*}, \nu^{*}\right)$ that needs to satisfy the following system of six equations: ${ }^{9}$

$$
\begin{gathered}
\rho+\sigma+\left[1-a_{1}(1-\varepsilon)\right] \nu=\gamma f_{K}\left(\gamma, \delta u \frac{h}{k}\right)+\frac{k}{h}\left[\frac{a_{2}}{a_{1}} \frac{1}{l} \frac{c}{k}-\frac{h}{k} \delta f_{L}\left(\gamma, \delta u \frac{h}{k}\right)\right] r\left(u, \frac{h}{k}\right) \\
\nu=\psi(1-l-s-u)-\theta \\
\rho-n=\psi(u+s)+a_{1}(1-\varepsilon) \nu-\frac{a_{3}}{a_{2}} \psi l \\
\frac{c}{k}=f\left(\gamma, \delta u \frac{h}{k}\right)-(\sigma+n+\nu) \\
s \frac{a_{2}}{a_{1}}=(1-\delta) g_{L}\left[(1-\gamma),(1-\delta) u \frac{h}{k}\right] \frac{a_{3} l-a_{2} s}{a_{1}}+s l \delta f_{L}\left(\gamma, \delta u \frac{h}{k}\right) \frac{h}{k} \frac{k}{c} \\
g\left[(1-\gamma),(1-\delta) u \frac{h}{k}\right]=s \frac{h}{k},
\end{gathered}
$$

where

$$
r\left(u, \frac{h}{k}\right)=\frac{(1-\gamma) g_{K}\left((1-\gamma),(1-\delta) u \frac{h}{k}\right)}{(1-\delta) g_{L}\left((1-\gamma),(1-\delta) u \frac{h}{k}\right)},
$$

is formally equivalent to quantity $R(u, k, h)$ defined by (34).

Appendix 6.3 also shows that conditions (40) and (44) can be rewritten in the alternative forms:

$$
\rho+\sigma+\left[1-a_{1}(1-\varepsilon)\right] \nu=\gamma f_{K}\left(\gamma, \delta u \frac{h}{k}\right)+\frac{k}{h} \frac{c}{k} \frac{\xi\left(u, \frac{h}{k}\right)}{u} \frac{a_{3} l-a_{2} s}{a_{1} l} r\left(u, \frac{h}{k}\right)
$$

\footnotetext{
${ }^{9}$ Note that, due to our assumptions on $f$ and $g$, partial derivatives $f_{K}, f_{L}, g_{K}$ and $g_{L}$ are homogeneous functions of degree zero such that, e.g. $f_{K}(\gamma k, \delta u h)=f_{K}\left(\gamma, \delta u \frac{h}{k}\right)$.
} 


$$
\frac{a_{2}}{a_{1}}=\frac{l \xi\left(u, \frac{h}{k}\right)}{u+s \xi\left(u, \frac{h}{k}\right)} \frac{a_{3}}{a_{1}}+\frac{u}{u+s \xi\left(u, \frac{h}{k}\right)} l \delta f_{L}\left(\gamma, \delta u \frac{h}{k}\right) \frac{h}{k} \frac{k}{c}
$$

where

$$
\xi\left(u, \frac{h}{k}\right)=1-\frac{(1-\gamma) g_{K}\left((1-\gamma),(1-\delta) u \frac{h}{k}\right)}{g\left[(1-\gamma),(1-\delta) u \frac{h}{k}\right]} .
$$

Generally speaking, system (40)-(45) may contain multiple interior solutions, as will be demonstrated in Section 4 using Cobb-Douglas production functions. Leaving this aside for the moment, the BGP conditions in the general form (40)-(45) can be used to carry out a comparison between different BGP solutions in the SE, as well as between the SE and the PE, in terms of dynamic efficiency. As mentioned above, we do this by adopting two different comparison criteria: a wealth criterion based on growth rates of output per capita, and a welfare criterion based on utility levels. According to the first criterion, we will compare the growth rate of output per capita at different BGP solutions ${ }^{10}$. According to the second criterion, we will compare discounted lifetime utility along BGPs. This will require specifying utility function $U$ in the standard form of CES utility (see Section 3)

These criteria will offer a broad view on the comparison across BGPs and across economies, PE and SE, in terms of wealth and welfare.

\subsection{Comparison across BGP equilibria in the SE}

Denote by $B G P_{A}$ and $B G P_{B}$ two BGPs of the SE and by $u^{A}, l^{A}, \lambda^{A}, s^{A}, \nu^{A}$ and $u^{B}, l^{B}, \lambda^{B}, s^{B}, \nu^{B}$ the corresponding time allocation levels (for labor, pure leisure, education, and consumption of services) and the growth rate, respectively. As discussed below, such BGPs need not necessarily be interpreted as multiple coexisting BGPs for a specified parameter selection, but also as a (possibly unique) BGP solution under two different parameter selections. The results summarized in the next Section follow from conditions (41)-(42) and establish a set of relations between time allocation, growth and social welfare at two different BGPs of the SE.

\subsubsection{Growth rates in the SE}

The following Proposition 1 provides a number of conditions concerning the growth rate differential in two BGPs of the SE.

\section{Proposition 1}

$$
\begin{gathered}
\nu^{B} \gtreqless \nu^{A} \Longleftrightarrow \lambda^{B} \gtreqless \lambda^{A} \\
\nu^{B} \gtreqless \nu^{A} \Longleftrightarrow l^{B} \lesseqgtr l^{A} \\
\text { for } 0<\varepsilon<1+\frac{a_{3}}{a_{1} a_{2}}, \nu^{B} \gtreqless \nu^{A} \Longleftrightarrow u^{B}+s^{B} \lesseqgtr u^{A}+s^{A} \Longleftrightarrow \lambda^{B}+l^{B} \gtreqless \lambda^{A}+l^{A} \\
\text { for } \varepsilon=1+\frac{a_{3}}{a_{1} a_{2}}, u^{B}+s^{B}=u^{A}+s^{A}, \quad \lambda^{B}+l^{B}=\lambda^{A}+l^{A} \\
\text { for } \varepsilon>1+\frac{a_{3}}{a_{1} a_{2}}, \nu^{B} \gtreqless \nu^{A} \Longleftrightarrow u^{B}+s^{B} \gtreqless u^{A}+s^{A} \Longleftrightarrow \lambda^{B}+l^{B} \lesseqgtr \lambda^{A}+l^{A}
\end{gathered}
$$

\footnotetext{
${ }^{10}$ In Section 3 we discuss how this comparison across different BGPs should be interpreted of the SE and (by regarding program (27) as special case of (26)) between the BGP dynamics of the SE and the PE
} 
Proof. See Appendix 6.4.

In particular, concerning parts (50) and (51) of Proposition 1, the proof given in Appendix 6.4 indicates that the growth rate differential $\nu^{B}-\nu^{A}$ is positively proportional to the differential of time spent on education, $\lambda^{B}-\lambda^{A}$, and negatively proportional to the differential of time spent on pure leisure, $l^{B}-l^{A}$. No similar clear-cut relation exists with labor time. However, part (52)-(54) establish relations between the growth rate differential and the differential in the time share $u+s$, which we may call 'uptime'. ${ }^{11}$ The direction of these relations strongly depends on the shape of the utility function. In this respect, the most common case in the literature is $0<\varepsilon \leq 1$, which is entirely included in part (52) of Proposition 1.

Interestingly, a broader interpretation can be given to Proposition 1. As mentioned above, the BGPs that are compared in Proposition 1 are not to be necessarily regarded as coexisting BGPs for a given parameter setting. Rather, they may represent the 'same' (possibly unique) BGP of the economy under different parameter settings (in particular, a different exogenous intersectoral allocation $\gamma, \delta$ of physical and human capital). In this second respect, although the coordinates of a BGP (in particular growth rate and time allocation) depend, in general, on all parameters of the model, the proof of Proposition 1 in Appendix 6.4 indicates that implications (50) and (54) are uniquely based on equations (41) and (42)), for fixed values of parameters $\psi, \theta, \rho, n$ and of those characterizing the utility function. In other words, the results in Proposition 1 are independent of parameters $\sigma$, of parameters $\gamma, \delta$ (exogenous intersectoral allocation) and of those (not yet specified) incorporated in the production functions, and are thus preserved under changes of such parameters. If, e.g. the growth rate at the (unique) BGP changes from level $\nu^{A}$ to level $\nu^{B}>\nu^{A}$ as a consequence of an exogenous change of intersectoral resource allocation $\gamma, \delta$, also the BGP levels of variables $u, l, \lambda, s$ will change according to the implications stated by Proposition 1 . This strengthens our results, that are necessarily based on exogenously assumed intersectoral allocation parameters $(\gamma$ and $\delta)$, due to the nature of our partial analysis.

Of course, a complete characterization of the dynamics of the SE across different BGP equilibria would require a deeper analysis of the relations between growth rate differentials and the other dynamic variables (output, the ratio of consumption to physical capital and of human capital to physical capital). This issue is successfully tackled by Ladrón-de Guevara et al. [1999] in the particular case of $\mathrm{PE}$, but no clear cut conditions about the role played by these variables can be obtained for the SE. However, for the sake of completeness, in Appendix 6.6 and 6.7 we provide a larger set of analytical conditions that may constitute the basis for dealing with such issues in the SE in the case of CobbDouglas technology. ${ }^{12}$ Moreover, by specializing such conditions to the PE, Appendix 6.7 offers an alternative proof and perspective on the comparative results obtained in Ladrón-de Guevara et al. [1999]. ${ }^{13}$

\subsubsection{Social welfare in the SE}

In a $\mathrm{BGP}^{14}$ per capita consumption $c^{*}(t)$ and capital $k^{*}(t)$ grow at the same rate $\nu^{*}$, and their ratio $(c / k)^{*}:=\chi^{*}$ is constant for any $t$. Therefore, from $c^{*}(t)=c_{0} \exp \left(\nu^{*} t\right), k^{*}(t)=k_{0} \exp \left(\nu^{*} t\right)$ one finally gets $c_{0}=\chi^{*} k_{0}$. The value of the objective functional of program (26) at a BGP with utility function

\footnotetext{
${ }^{11}$ We may regard $(u+s)$ as uptime since it is the portion of time having an immediate and direct effect on the output of the two sectors at time $t$ and, conversely, we may define $(\lambda+l)$ as 'downtime'.

${ }^{12}$ Such conditions also provide insight on why the interplay between the dynamic variables at the BGPs is much more complicated in the SE than in the PE.

${ }^{13}$ See Proposition 4.2 in Ladrón-de Guevara et al. [1999], which is basically proven via implicit function differentiation based on the system of equations defining the BGP solutions. In contrast, the proof provided in Appendix 6.7 is entirely based on a chain of conditional implications from a set of inequalities.

${ }^{14}$ In the first part of this Section, an asterisk denotes levels and trajectories of the dynamic variables in a generic BGP.
} 
(38), given by

$$
V^{*}=\int_{0}^{\infty} \frac{1}{1-\varepsilon}\left[\left(c^{*}(t)\right)^{a_{1}}\left(l^{*}\right)^{a_{2}}\left(s^{*}\right)^{a_{3}}\right]^{1-\varepsilon} \exp [(n-\rho)] t d t
$$

can thus be rewritten as a function of variables $l, s, \chi:=(c / k)$ and $\nu$, as follows:

$$
V^{*}=\frac{1}{1-\varepsilon}\left[\left(k_{0} \chi^{*}\right)^{a_{1}}\left(l^{*}\right)^{a_{2}}\left(s^{*}\right)^{a_{3}}\right]^{1-\varepsilon} \int_{0}^{\infty} \exp \left\{\left[(n-\rho)+a_{1}(1-\varepsilon) \nu^{*}\right] t\right\} d t,
$$

provided that the convergence condition $a_{1}(1-\varepsilon) \nu^{*}<\rho-n$ is satisfied. Under the standard assumption $\rho-n>0$, convergence holds for any $\varepsilon \geq 1$, whereas in the case $0<\varepsilon<1$ a further parameter restriction is required for convergence, namely, $\nu^{*}<(\rho-n) /\left[a_{1}(1-\varepsilon)\right]$. Finally, discounted lifetime utility in the $\mathrm{SE}$ is expressed as:

$$
\begin{gathered}
V^{*}=\frac{1}{1-\varepsilon}\left[\left(k_{0} \chi^{*}\right)^{a_{1}}\left(l^{*}\right)^{a_{2}}\left(s^{*}\right)^{a_{3}}\right]^{1-\varepsilon} \frac{1}{(\rho-n)-a_{1}(1-\varepsilon) \nu^{*}} \quad \varepsilon \neq 1 \\
V^{*}=\frac{1}{\rho-n}\left[a_{1} \ln \left(k_{0} \chi^{*}\right)+a_{2} \ln l^{*}+a_{3} \ln s^{*}\right] \quad \varepsilon=1 .
\end{gathered}
$$

Now consider two BGPs of the SE, say $B G P_{A}$ and $B G P_{B}$ (with variables denoted by different subscripts). Define $\Gamma(\nu):=\left[(\rho-n)-a_{1}(1-\varepsilon) \nu\right]^{-1}$, which is easily checked to be an increasing (respectively decreasing) function of $\nu$ for $0<\varepsilon<1$ (resp. $\varepsilon>1$ ). For given $k_{0}$ define also $Q(\chi, l, s):=\frac{1}{1-\varepsilon}\left[\left(k_{0} \chi\right)^{a_{1}} l^{a_{2}} s^{a_{3}}\right]^{1-\varepsilon}$. In particular, assume $0<\varepsilon<1$. Since $\nu^{B}>\nu^{A}$ implies both $\Gamma\left(\nu^{B}\right)>\Gamma\left(\nu^{A}\right)$ and $l^{B}<l^{A}$ (where the latter follows from Proposition 1), we have no clear indications on whether a positive growth rate differential $\nu^{B}-\nu^{A}$ will be associated or not with a utility differential $\left(V^{B}-V^{A}\right)$ of the same sign, in general. Moreover, note that quantity $Q(\chi, l, s)$ depends on the BGP value of $s$ and on that of $\chi=c / k$. Inspection of (43) reveals that the latter may depend crucially on exogenous parameters $\gamma$ and $\delta$. Therefore, one can easily figure out situations in which $\nu^{B}>\nu^{A}$ but $V^{B}<V^{A}$, or vice versa. As a limiting case, discounted log-utility (57) does not depend on the growth rate explicitly.

\subsection{BGP conditions for the PE}

Before carrying out a comparison between the BGP properties of the SE and the PE, in this Section we list the BGP conditions for the model investigated in Ladrón-de Guevara et al. [1999] ${ }^{15}$, which we denote as PE in our framework. Such conditions can be either directly obtained by solving the program (27), with the following CES utility function

$$
U(c, l)=\frac{1}{1-\varepsilon}\left(c^{a} l^{1-a}\right)^{1-\varepsilon},
$$

or by imposing suitable restrictions to conditions (40)-(45). More precisely, we consider a limiting case where $a_{3} \rightarrow 0$ and consequently $s \rightarrow 0$. Consistently, we also assume $\gamma, \delta \rightarrow 1$ (see Section 2.3 for details). The BGP conditions become in this case:

$$
\begin{gathered}
\rho+\sigma+[1-a(1-\varepsilon)] \nu=f_{K}\left(1, u \frac{h}{k}\right) \\
\nu=\psi(1-l-u)-\theta
\end{gathered}
$$

\footnotetext{
${ }^{15}$ See the case of constant returns to scale in the education sector, in Ladrón-de Guevara et al. [1999].
} 


$$
\begin{gathered}
\rho-n=\psi u+a(1-\varepsilon) \nu \\
\frac{c}{k}=f\left(1, u \frac{h}{k}\right)-(\sigma+n+\nu) \\
\frac{1-a}{a}=l f_{L}\left(1, u \frac{h}{k}\right) \frac{h}{k} \frac{k}{c}
\end{gathered}
$$

resulting in a system of 5 equation in 5 variables $((c / k),(h / k), l, u, \nu)$. Note that conditions $(59)$ and (63) are highly simplified as compared with their more general counterparts (40) and (44), respectively. The final part of Appendix 6.3 provides some details about such simplifications. A comparison between the BGP conditions of the two economies is carried out in the next Section.

\subsection{Comparison of BGP equilibria in the $\mathrm{SE}$ and $\mathrm{PE}$}

Our comparison between BGP equilibria in the PE and the SE relies again on two complementary criteria. The first criterion considers the growth rate of output per capita in a BGP, the second criterion considers discounted lifetime utility in a BGP. Both comparison exercises are carried out by assuming that parameters $\psi, \theta, \rho, n, \varepsilon$ are identical across the two economies. We also assume that the utility share of consumption is the same in the two economies, $a_{1}=a$, whereas utility share $1-a$ of leisure time is splitted into $a_{2}+a_{3}=1-a_{1}$ when switching from PE to SE. ${ }^{16}$ Finally, we denote by $u^{S E}, l^{S E}, \lambda^{S E}, s, \nu^{S E}$ and $u^{P E}, l^{P E}, \lambda^{P E}, \nu^{P E}$ the time allocation variables (labor, pure leisure, education, services consumption) and the growth rate in a BGP of the SE and the PE, respectively. The results stated in the next two subsections establish a set of relations between the BGPs of the two economies, in terms of time allocation, growth and social welfare, assuming that the PE and the $\mathrm{SE}$ are otherwise characterized by identical parameters.

\subsubsection{Growth rates: SE vs. PE}

The following Proposition 2 can be proven, which provides (necessary and/or sufficient) conditions for the growth rate of the SE to be larger than that of the PE.

\section{Proposition 2}

$$
\begin{gathered}
\nu^{S E} \gtreqless \nu^{P E} \Longleftrightarrow \lambda^{S E} \gtreqless \lambda^{P E} \\
\text { for } 0<\varepsilon<1, u^{S E}+s \leq u^{P E} \Longrightarrow \nu^{S E}>\nu^{P E} \\
\nu^{S E}>\nu^{P E} \Longrightarrow l^{S E}<l^{P E}
\end{gathered}
$$

Proof. See Appendix 6.5.

The results stated by Proposition 2 are quite general and apply to any pair of BGPs from the SE and the PE, respectively. ${ }^{17}$ In particular, according to (64), the growth rate differential has the same sign of the educational differential. More precisely, as shown in Appendix 6.5, they are proportional to each other. From (65), for $0<\varepsilon<1$, a sufficient condition for higher growth in the SE is that the time share spent on service consumption $(s)$ does not exceed the differential in labor time $u^{P E}-u^{S E}$. According to (66), a positive growth differential requires a negative differential in the time share spent on pure leisure activities. Note also that, for $0<\varepsilon<1, u^{S E}<u^{P E}$ is not per se enough to attain higher growth in the $\mathrm{SE}$, nor is reducing both leisure time and labor time $\left(l^{S E}<l^{P E}, u^{S E}<u^{P E}\right)$ sufficient for this. Put differently, Proposition 2 can be interpreted as follows. Reallocating time (from

\footnotetext{
${ }^{16}$ We remember that in PE $s \equiv 0$ and consequently $\gamma=\delta=1$.

${ }^{17}$ See the next Section for examples with multiple BGPs.
} 
the PE to the SE) in such a way that $l^{S E}<l^{P E}$ and $u^{S E}<u^{P E}$ necessary implies spending more time in the sum of educational activities and service consumption activities (that is, $\lambda^{S E}+s>\lambda^{P E}$ ). Therefore, higher growth necessarily requires that the time saved from work and pure leisure is not just reallocated to service consumption (in such a way that $\lambda^{S E}+s>\lambda^{P E}$ ), but a larger fraction of time spent on education is also necessary (and sufficient). We remark that (64) and (66) hold for any $\varepsilon>0$, whereas $(65)$ holds for $0<\varepsilon<1$. $^{18}$

\subsubsection{Social welfare: SE vs. PE}

In this case the value of the objective functional at a BGP of the SE, given by (56)-(57) is compared with its counterpart for the PE, namely:

$$
\begin{gathered}
V^{*}=\frac{1}{1-\varepsilon}\left[\left(k_{0} \chi^{*}\right)^{a_{1}}\left(l^{*}\right)^{1-a_{1}}\right]^{1-\varepsilon} \frac{1}{(\rho-n)-a_{1}(1-\varepsilon) \nu^{*}} \quad \varepsilon \neq 1 \\
V^{*}=\frac{1}{\rho-n}\left[a_{1} \ln \left(k_{0} \chi^{*}\right)+\left(1-a_{1}\right) \ln l^{*}\right] \quad \varepsilon=1 .
\end{gathered}
$$

In addition to quantities $\Gamma(\nu)$ and $Q(\chi, l, s)$, defined in Section 3.2.2, the following definition is also useful: $Q(\chi, l, 0):=\frac{1}{1-\varepsilon}\left[\left(k_{0} \chi\right)^{a_{1}} l^{1-a_{1}}\right]^{1-\varepsilon}$. In particular, we assume $0<\varepsilon<1$. Again, it is immediate to check that $\nu^{S E}>\nu^{P E}$ implies $\Gamma\left(\nu^{S E}\right)>\Gamma\left(\nu^{P E}\right)$. From Proposition 2 (see (66)) and the fact that $1-a_{1}=a_{2}$ in the $\mathrm{PE}, \nu^{S E}>\nu^{P E}$ also implies $\left(l^{S E}\right)^{a_{2}}<\left(l^{P E}\right)^{1-a_{1}}$. More generally, quantities $Q(\chi, l, s)$ and $Q(\chi, l, 0)$ depend on the equilibrium value of $s$ (in the SE) and on that of $\chi=c / k$ (in both economies). As pointed out in Section 3.2.2, the latter depends crucially on exogenous parameters $\gamma$ and $\delta$ in the SE. Therefore, there is no clear association between the sign of the utility differential $\left(V^{S E}-V^{P E}\right)$ and that of the growth differential $\left(\nu^{S E}>\nu^{P E}\right)$. Again, one can easily figure out situations in which $\nu^{S E}>\nu^{P E}$ but $V^{S E}<V^{P E}$, or vice versa.

\section{Computation of the BGP under Cobb-Douglas technology}

In this Section we show how system (40)-(45) can be solved in the case of Cobb-Douglas technology, and provide evidence of the existence of multiple BGPs for a wide region of the parameter space. The general idea on how to deal with the system of BGP conditions is based on Ladrón-de Guevara et al. [1999], who solve a particular and lower-dimensional case of the present model corresponding to program (27) for the PE. What is demonstrated below represents in fact a generalization of the solution strategy adopted by Ladrón-de Guevara et al. [1999]. Assume constant-returns-to-scale Cobb-Douglas technology for both the industrial and the service sector, namely:

$$
\begin{gathered}
f\left(\gamma, \delta u \frac{h}{k}\right)=B \gamma^{\beta}\left(\delta u \frac{h}{k}\right)^{1-\beta} \\
g\left((1-\gamma),(1-\delta) u \frac{h}{k}\right)=C(1-\gamma)^{\eta}\left((1-\delta) u \frac{h}{k}\right)^{1-\eta}
\end{gathered}
$$

with $B, C>0,0<\beta<1,0<\eta<1$. As shown in the Appendix 6.6, the assumption of Cobb-Douglas technologies simplifies system (40)-(45) remarkably, mainly because marginal products $f_{K}, f_{L}, g_{K}, g_{L}$ can easily be rewritten in terms of $f$ and $g$, respectively. Note also that, for any given $u=\bar{u}>0$, quantity $f\left(\gamma, \delta u \frac{h}{k}\right):=\Phi=m(u h / k)$ is a monotonically increasing function of $(h / k)$. Conversely,

\footnotetext{
${ }^{18}$ The case $0<\varepsilon<1$ is the one theoretical and empirical literature cares most about.
} 
$(h / k)=(1 / u) m^{-1}(\Phi)$. Therefore, further simplification arises from replacing variable $(h / k)$ with the new variable $\Phi$, when it comes to solve partial system (40)-(43) for a given initial choice of $(\bar{l}, \bar{s})$. With such simplifications, equations (40), (43), (44), (45) are rewritten, respectively, as:

$$
\begin{gathered}
\rho+\sigma+\left[1-a_{1}(1-\varepsilon)\right] \nu=\beta \Phi+\left[\frac{a_{2}}{a_{1}} \frac{1}{l} \frac{c}{k}-\frac{1-\beta}{u} \Phi\right] \frac{\eta}{1-\eta} u \\
\frac{c}{k}=\Phi-(\sigma+n+\nu) \\
(1-\eta) \frac{1}{u} \frac{a_{3} l-a_{2} s}{a_{1}}+l \frac{1-\beta}{u} \Phi \frac{k}{c}=\frac{a_{2}}{a_{1}} \\
g\left((1-\gamma),(1-\delta) m^{-1}(\Phi)\right)-\frac{s}{u} m^{-1}(\Phi)=0
\end{gathered}
$$

where

$$
m^{-1}(\Phi)=\frac{1}{\delta}\left(\frac{\Phi}{B \gamma^{\beta}}\right)^{\frac{1}{1-\beta}}, \quad g(x, y)=C x^{\eta} y^{1-\eta},
$$

whereas (41), (42) remain unchanged.

Moreover, as shown in Appendix 6.6, (71) and (73) can be rewritten in alternative forms, respectively as follows:

$$
\begin{gathered}
\rho+\sigma+\left[1-a_{1}(1-\varepsilon)\right] \nu=\beta \Phi+\eta \frac{c}{k} \frac{a_{3} l-a_{2} s}{a_{1}} \\
\frac{l}{u+(1-\eta) s}\left[(1-\eta) \frac{a_{3}}{a_{1}}+(1-\beta) \Phi \frac{k}{c}\right]=\frac{a_{2}}{a_{1}} .
\end{gathered}
$$

Broadly speaking, for given $\bar{l}$ and $\bar{s}$ (such that $0<\bar{l}, \bar{s}<1, \bar{l}+\bar{s}<1$ ), equations (41) and (42) define $\bar{u}$ and $\bar{\nu}$ as simple functions of $\bar{l}$ and $\bar{s}$. Next, equations (71) and (72) define explicitly, via $\bar{u}$ and $\bar{\nu}$, variables $\Phi$ and $(c / k)$ as functions of $\bar{l}$ and $\bar{s}$, too. Finally, substitution into equations (73)-(74) yields a nonlinear system of two equations in the unknowns $(\bar{l}, \bar{s})$, which can then be solved numerically.

More precisely, note that system of equations (71), (41), (42), (72) admits a unique (and explicit) solution $(\bar{u}, \bar{\nu},(\overline{c / k}), \bar{\Phi})$ for any arbitrary $(\bar{l}, \bar{s})$ within a suitable range. Given $(\bar{l}, \bar{s})$, the system of the two equations (41)-(42) is linear in $u, \nu$, yielding solution

$$
\begin{gathered}
\bar{\nu}=\frac{1}{\tau}\left[\psi\left(1-\frac{a_{2}+a_{3}}{a_{2}} \bar{l}\right)-\theta-(\rho-n)\right]:=\nu(\bar{l}) \\
\bar{u}=1-\bar{l}-\bar{s}-\frac{\theta}{\psi}-\frac{1}{\psi} \nu(\bar{l}):=u(\bar{l}, \bar{s}),
\end{gathered}
$$

where $\tau:=1-a_{1}(1-\varepsilon)$, with $\tau=1$ for $\varepsilon=1$ (log-utility) and $\tau \lessgtr 1$ for $\varepsilon \lessgtr 1$. Next, by replacing (72) into (71) one obtains: ${ }^{19}$

$$
\bar{\Phi}=\frac{\rho+\sigma+\tau \nu(\bar{l})+(\sigma+n+\nu(\bar{l})) \frac{a_{2}}{a_{1}} \frac{u(\bar{l}, \bar{s})}{\bar{l}} \frac{\eta}{1-\eta}}{\beta+\frac{\eta}{1-\eta}\left[\frac{a_{2}}{a_{1}} \frac{u(\bar{l}, \bar{s})}{\bar{l}}-(1-\beta)\right]}:=\Phi(\bar{l}, \bar{s})
$$

\footnotetext{
${ }^{19}$ Equivalent formulas for $\bar{\Phi}$ and $\overline{\left(\frac{c}{k}\right)}$ can be obtained (which we do not report here) using BGP conditions (75) and (76) instead of (71) and (73).
} 


$$
\overline{\left(\frac{c}{k}\right)}=\frac{\rho+\sigma+\tau \nu(\bar{l})-(\sigma+n+\nu(\bar{l}))\left[\beta-(1-\beta) \frac{\eta}{1-\eta}\right]}{\beta+\frac{\eta}{1-\eta}\left[\frac{a_{2}}{a_{1}} \frac{u(\bar{l}, \bar{s})}{\bar{l}}-(1-\beta)\right]}:=\left(\frac{c}{k}\right)(\bar{l}, \bar{s}) .
$$

Finally, by substituting the above determined quantities $(\bar{u}, \bar{\nu},(\overline{c / k}), \bar{\Phi})$ into conditions (73)-(74), and then denoting their left-hand sides by $\Psi(\bar{l}, \bar{s})$ and $\Xi(\bar{l}, \bar{s})$, respectively, the BGPs are determined numerically as the solutions of

$$
\left\{\begin{array}{c}
\Psi(\bar{l}, \bar{s})=\frac{a_{2}}{a_{1}} \\
\Xi(\bar{l}, \bar{s})=0
\end{array}\right.
$$

provided that all the required feasibility constraints are satisfied on the control variables $\bar{l}, \bar{u}, \bar{s}$, and on the ratios $\overline{\left(\frac{c}{k}\right)}$ and $\overline{\left(\frac{h}{k}\right)}$ (or, equivalently, $\left.\bar{\Phi}\right) \cdot{ }^{20}$

We provide below a numerical example showing the possibility of multiple BGPs for plausible ranges of the parameters. We adopt the baseline parameter setting of Ladrón-de Guevara et al. [1999], namely $\rho=0.05, n=0, \sigma=0, \theta=0, \psi=0.23, B=1$. Parameters $a=a_{1}, \beta$ and $\varepsilon$ are allowed to vary within suitable ranges in Ladrón-de Guevara et al. [1999]. In particular, we select $a=a_{1}=0.3, \beta=0.36$ and $\varepsilon=0.88$, a situation in which Ladrón-de Guevara et al. [1999] find three coexisting BGPs in the Post-Industrial Economy (corresponding to the particular case $a_{3}=0$, $a_{2}=1-a_{1}=0.7, \gamma=\delta=1$ in our model, which results in $\left.s^{*}=0\right)$. The coordinates of the three coexisting BGPs are reported in Table 1 (where $\lambda^{*}=1-l^{*}-s^{*}-u^{*}$ ), along with the maximized utility $V^{*}$ at each BGP.

\begin{tabular}{l|llllllll}
\hline \hline & $l^{*}$ & $s^{*}$ & $u^{*}$ & $\lambda^{*}$ & $\nu^{*}$ & $(c / k)^{*}$ & $(h / k)^{*}$ & $V^{*}$ \\
\hline BGP1 & 0.706 & 0 & 0.215 & 0.079 & 0.0182 & 0.169 & 0.341 & 153.87 \\
BGP2 & 0.752 & 0 & 0.216 & 0.032 & 0.0074 & 0.151 & 0.261 & 152.84 \\
BGP3 & 0.783 & 0 & 0.217 & 0 & 0 & 0.138 & 0.208 & 152.03 \\
\hline \hline
\end{tabular}

Table 1: multiple BGPs in the PE.

BGP1 and BGP2 are interior steady states, whereas BGP3 is a non-interior steady state, with no time allocated to education and zero growth. Moreover, for similar parametrizations, Ladrón-de Guevara et al. [1999] find that stationary solutions BGP1 and BGP3 are both saddle-path stable, implying that the economy may converge to one or the other depending on the initial condition. When $\varepsilon$ is sufficiently close to 1 , the range of $\beta$ for which the economy displays multiple steady states (the other parameters being equal to their baseline values) is limited: smaller values of $\beta$ result in just one interior steady state, whereas one non interior steady state exists for larger values of $\beta$.

We now introduce service consumption and a service sector accordingly. We assume $C=1$ and $\eta=0.25$ as the parameters of the Cobb-Douglas production function for leisure services. We also assume $a_{3}=0.03$ and $a_{2}=1-a_{1}-a_{3}=0.67$. Accordingly, shares $\gamma$ and $\delta$ of resources allocated to the industrial sector are reduced from 1 to 0.92. Table 2 shows that the BGP structure observed in the $\mathrm{PE}$ for the baseline parametrization is preserved under transition to the SE:

\footnotetext{
${ }^{20}$ Obviously, the BGP solutions computed with this procedure are feasible if $0 \leq \bar{l}, \bar{u}, \bar{s} \leq 1, \bar{l}+\bar{u}+\bar{s} \leq 1$ and $\overline{\left(\frac{c}{k}\right)}, \bar{\Phi} \geq 0$, whereas an interior solution will be characterized, in particular, by $0<\bar{l}, \bar{u}, \bar{s}<1, \bar{l}+\bar{u}+\bar{s}<1$. Although it is possible to provide analytical conditions for an interior BGP, they are rather cumbersome to deal with.
} 


\begin{tabular}{l|llllllll}
\hline \hline & $l^{*}$ & $s^{*}$ & $u^{*}$ & $\lambda^{*}$ & $\nu^{*}$ & $(c / k)^{*}$ & $(h / k)^{*}$ & $V^{*}$ \\
\hline BGP1 & 0.670 & 0.031 & 0.213 & 0.085 & 0.0196 & 0.172 & 0.406 & 151.76 \\
BGP2 & 0.727 & 0.034 & 0.215 & 0.024 & 0.0056 & 0.149 & 0.287 & 150.46 \\
BGP3 & 0.749 & 0.036 & 0.215 & 0 & 0 & 0.139 & 0.242 & 149.86 \\
\hline \hline
\end{tabular}

Table 2: multiple BGPs in the SE for $a_{3}=0.03, \gamma=\delta=0.92$.

Comparison of Table 2 (SE) with Table 1 (PE) shows that higher growth may be associated with lower welfare (in particular, this is the case of BGP1). Also, as expected by our analytical results, higher growth is associated with higher education share and lower time shares absorbed by work and pure leisure.

Similar considerations can be drawn from a comparison between Table 1 and Table 3, which describes an economy where the service sector has a larger impact $\left(a_{3}=0.05, \gamma=\delta=0.86\right)$.

\begin{tabular}{l|llllllll}
\hline \hline & $l^{*}$ & $s^{*}$ & $u^{*}$ & $\lambda^{*}$ & $\nu^{*}$ & $(c / k)^{*}$ & $(h / k)^{*}$ & $V^{*}$ \\
\hline BGP1 & 0.645 & 0.053 & 0.212 & 0.091 & 0.0209 & 0.175 & 0.471 & 150.90 \\
BGP2 & 0.711 & 0.059 & 0.213 & 0.017 & 0.0039 & 0.147 & 0.309 & 149.33 \\
BGP3 & 0.727 & 0.061 & 0.213 & 0 & 0 & 0.140 & 0.275 & 148.92 \\
\hline \hline
\end{tabular}

Table 3: multiple BGPs in the SE for $a_{3}=0.05, \gamma=\delta=0.86$.

Again, the multiple steady-state structure is preserved, but numerical simulation reveals that this structure disappears for sufficiently large deviations from the baseline parametrization. In addition, comparison of Table 2 with Table 3 shows that a tradeoff between growth and welfare can be observed under parameter changes affecting the SE economy, as well.

More numerical experiments, highlighting the BGP structure and its impact on welfare and growth under alternative parametrizations, are available upon request.

\section{Conclusion}

In this paper, we propose a novel three-sector growth model, describing the joint dynamics of the industrial sector and the service sector and that of the education sector. Our paper generalizes the time allocation problem and, as a consequence, the extensions of the Uzawa-Lucas model dealing with leisure can be regarded as particular cases of our general model. In particular, the model of Ladrón-de Guevara et al. [1999] corresponds to an economic system without service sector, which we interpret as Post Industrial Economy (PE), while our full model corresponds to an economic system with service sector, which we define Service Economy (SE). The novelty of our results is mainly based on the way time left from work is allocated among different activities. In the PE total available time is allocated between work, leisure and education, while in the SE a portion of leisure time is absorbed by consumption of services - which represents a time-consuming activity - and only the rest is spent as purely free time. Thus, in the SE total available time is allocated between work, leisure and educational time, and in turn leisure is allocated between free time and service consumption time.

With the addition of a service production sector, our general model of the SE displays a complete 'taxonomy' of sectors and activities according to their effects on intertemporal equilibrium: i) equilibrium in the industrial sector entails an immediate effect, via the consumption flow at the time of production, as well as the intertemporal accumulation of the stock of physical capital; ii) the educational activity involves a purely intertemporal effect, since the stock of knowledge can only be accumulated to increase human capital; iii) finally, equilibrium in the service sector can be attained solely through consumption at the same time of production, since leisure services cannot be stored over time. 
We derive the BGP equilibria for the SE. Similarly to the model studied by Ladrón-de Guevara et al. [1999], we show that multiple BGPs are a possible outcome for realistic ranges of the parameters. In order to highlight the impact of time allocation on both growth and social welfare of the economy, we compare the BGP equilibria of the SE and PE, as well as different equilibria of the SE. The relationships between growth and time allocation in the BGP solutions are proven analytically. Moreover, analytical expressions for social welfare at the BGP solutions of the SE and the PE are derived, showing that larger growth is not necessarily mirrored by larger welfare in different solution paths. We thus argue that a satisfactory assessment of the implications of the transition between the PE and the SE - as well between different BGPs in the SE - should rely upon two complementary criteria: a wealth criterion based on the growth rate of output per capita, and a social criterion which considers discounted lifetime utility. In particular, Propositions 1 and 2 summarize the main analytical results and establish a set of relations between time allocation and growth at two different BGPs of the SE and at the BGPs of the SE and the PE, respectively, assuming that the PE and the SE are otherwise characterized by identical parameters. Our results are quite general, as they apply to both the case of multiple BGPs in the phase space and the case of a unique BGP under different parameter constellations. In particular, the BGPs that are compared in Proposition 1 may as well be interpreted as the 'same' (possibly unique) BGP of the economy under different parameter settings. On the other hand, the results stated by Proposition 2 are quite general and apply to any pair of BGPs from the SE and the $\mathrm{PE}$, respectively. The results of the comparison summarized by Proposition 1 indicate that the growth rate differential in two BGPs of the SE is positively proportional to the differential of time spent on education and negatively proportional to the differential of time spent on pure leisure. Similar relations - the sign of which depends on the CES utility parameter - exist between growth rate and the aggregate time spent on labor and service consumption (which we call 'uptime'), although no clear-cut relations exist between growth and labor time in isolation. Turning to the comparison between SE and $\mathrm{PE}$, Proposition 2 shows that the growth rate differential is again proportional to the educational differential. For $0<\varepsilon<1$, a sufficient condition for higher growth in the SE than in the PE is that the time share spent on service consumption does not exceed that saved from reducing labor time. Moreover, higher growth in the SE cannot be achieved without reducing the time share spent on pure leisure activities when switching from PE to SE.

From Propositions 1 and 2 we can draw the following conclusions and policy implications. In the SE the relation between rate of growth and 'uptime' strongly depends on the shape of the utility function. This result generalizes the relations between growth and labor time highlighted in Ladrón-de Guevara et al. [1999] for the case of the PE. Reallocating time from the PE to the SE (with a strictly positive time share spent on service consumption) in such a way that less time is absorbed by pure leisure is necessary but not sufficient to attain higher growth in the SE. Instead, as usual in growth models, higher growth is characterized by more time spent on educational activities. In general, in the SE this condition can be achieved through a larger range of possible tradeoffs than in the PE, as suggested by Proposition 1. Furthermore, Proposition 2 indicates that a typical situation compatible with higher growth in the $\mathrm{SE}$ than in the $\mathrm{PE}$ (in the case $0<\varepsilon<1$, most often assumed in the literature) is one with a larger share of education time, and smaller shares of pure leisure and work time. Finally, as Sections 3.2.2 and 3.4.2 suggest, and numerical experiments confirm, intersectoral resource allocation (exogenously assumed in our analysis) turns out to be important in governing the tradeoffs between growth and social welfare. This may represent an important issue from a policy-making perspective. 


\section{Appendix}

\subsection{General first-order optimality conditions}

Here we derive the general first-order conditions for program (26) with utility $U(c, l, s)$, continuous, twice differentiable, strictly concave and increasing in $c, l, s$, with technologies $f$ and $g$ homogeneous of degree one. The current-value Hamiltonian of program (26) is given by:

$$
\begin{aligned}
H(c, l, u, s, k, h) & =U(c, l, s)+\pi_{1}[f(\gamma k, \delta u h)-c-(\sigma+n) k]+\pi_{2}[\psi(1-l-s-u) h-\theta h] \\
& +\pi_{3}\{g[(1-\gamma) k,(1-\delta) u h]-s h\} .
\end{aligned}
$$

Conditions (28)-(31) correspond to the stationarity conditions of $H$ with respect to the control variables $c, l, u, s$, respectively. Based on the Maximum Principle, the general dynamic conditions on the costate variables:

$$
\dot{\pi}_{1}-(\rho-n) \pi_{1}=-\frac{\partial H}{\partial k}, \quad \dot{\pi}_{2}-(\rho-n) \pi_{2}=-\frac{\partial H}{\partial h}
$$

correspond, respectively, to

$$
\begin{aligned}
\frac{\dot{\pi}_{1}}{\pi_{1}}=\rho+ & \sigma-\gamma f_{K}(\gamma k, \delta u h)-\frac{\pi_{3}}{\pi_{1}}(1-\gamma) g_{K}[(1-\gamma) k,(1-\delta) u h] \\
\frac{\dot{\pi}_{2}}{\pi_{2}} & =\rho-n+\theta-\psi(1-l-s-u)-\frac{\pi_{1}}{\pi_{2}} \delta u f_{L}(\gamma k, \delta u h)+ \\
& -\frac{\pi_{3}}{\pi_{2}}\left\{(1-\delta) u g_{L}[(1-\gamma) k,(1-\delta) u h]-s\right\}
\end{aligned}
$$

Consider condition (79) first. Note that it follows from condition (30) that:

$$
\pi_{1} \delta u f_{L}(\gamma k, \delta u h)+\pi_{3}(1-\delta) u g_{L}((1-\gamma) k,(1-\delta) u h)=\pi_{2} u \psi .
$$

Substituting into (79), the latter simplifies into:

$$
\frac{\dot{\pi}_{2}}{\pi_{2}}=\rho-n+\theta-\psi(1-l-s)+\frac{\pi_{3}}{\pi_{2}} s .
$$

Moreover, from (29) and (31) one obtains:

$$
s \frac{U_{s}(c, l, s)}{U_{l}(c, l, s)}=s+\frac{\pi_{3}}{\pi_{2}} \frac{1}{\psi} s,
$$

and therefore

$$
s \frac{\pi_{3}}{\pi_{2}}=\psi\left[s \frac{U_{s}(c, l, s)}{U_{l}(c, l, s)}-s\right] .
$$

Finally, substitution into (81) yields (33).

Consider now condition (78). Similarly to the previous case, from (29) and (28) one obtains:

$$
\frac{\pi_{2}}{\pi_{1}}=\frac{U_{l}(c, l, s)}{h U_{c}(c, l, s) \psi},
$$

whereas (80) can be rewritten as

$$
\frac{\pi_{2}}{\pi_{1}}=\frac{\delta f_{L}(\gamma k, \delta u h)}{\psi}+\frac{\pi_{3}}{\pi_{1}} \frac{(1-\delta) g_{L}[(1-\gamma) k,(1-\delta) u h]}{\psi} .
$$


Therefore, by equating the right-hand sides of the latter two equations, one obtains

$$
\frac{U_{l}(c, l, s)}{h U_{c}(c, l, s)}=\delta f_{L}(\gamma k, \delta u h)+\frac{\pi_{3}}{\pi_{1}}(1-\delta) g_{L}[(1-\gamma) k,(1-\delta) u h]
$$

from which:

$$
(1-\gamma) g_{K}[(1-\gamma) k,(1-\delta) u h] \frac{\pi_{3}}{\pi_{1}}=\frac{1}{h}\left[\frac{U_{l}(c, l, s)}{U_{c}(c, l, s)}-h \delta f_{L}(\gamma k, \delta u h)\right] R(u, k, h),
$$

where $R(u, k, h)$ is given by (34). Finally, substitution of the latter quantity into (78) results into (32).

\subsection{First-order conditions with CES utility}

Here we specialize the first-order conditions of program (26) to the case of CES utility according to (38) and, in particular, of log-utility according to (39). As is well known, a remarkable simplification is due to the fact that for the CES function:

$$
U=\frac{1}{1-\varepsilon}\left(\prod_{i=1}^{I} x_{i}^{a_{i}}\right)^{1-\varepsilon}, \quad \sum_{i=1}^{I} a_{i}=1, \quad \varepsilon \neq 1
$$

marginal utilities can be expressed as

$$
U_{i}=\frac{a_{i}}{x_{i}}\left(\prod_{i=1}^{I} x_{i}^{a_{i}}\right)^{1-\varepsilon}, \quad i=1,2, \ldots, I
$$

and therefore

$$
\frac{U_{i}}{U_{j}}=\frac{a_{i}}{a_{j}} \frac{x_{j}}{x_{i}}, \quad i, j=1,2, \ldots, I,
$$

where the latter two equations hold for the case of log-utility, too $(\varepsilon \rightarrow 1)$.

Therefore, for any $\varepsilon>0$, conditions (28)-(33) read, respectively:

$$
\begin{gathered}
\frac{a_{1}}{c}\left(c^{a_{1}} l^{a_{2}} s^{a_{3}}\right)^{1-\varepsilon}=\pi_{1} \\
\frac{a_{2}}{l}\left(c^{a_{1}} l^{a_{2}} s^{a_{3}}\right)^{1-\varepsilon}=\psi h \pi_{2} \\
\delta \pi_{1} f_{L}(\gamma k, \delta u h)+(1-\delta) \pi_{3} g_{L}((1-\gamma) k,(1-\delta) u h)=\psi \pi_{2} \\
a_{3}\left(c^{a_{1}} l^{a_{2}} s^{a_{3}}\right)^{1-\varepsilon}=s\left(\psi h \pi_{2}+h \pi_{3}\right) \\
\frac{\dot{\pi}_{1}}{\pi_{1}}=\rho+\sigma-\gamma f_{K}(\gamma k, \delta u h)-\frac{1}{h}\left[\frac{a_{2}}{a_{1}} \frac{c}{l}-h \delta f_{L}(\gamma k, \delta u h)\right] R(u, k, h) \\
\frac{\dot{\pi}_{2}}{\pi_{2}}=(\rho-n)+\theta-\psi(1-l)+\psi \frac{a_{3}}{a_{2}} l,
\end{gathered}
$$

where $R(u, k, h)$ is again specified as $(34)$. 


\subsection{Derivation of the BGPs}

A BGP solution is generally characterized as follows. Along a BGP $\dot{c} / c=\dot{k} / k=\dot{h} / h:=\nu$. In addition, $l, u$ and $s$ must remain constant (such that $\dot{l}, \dot{u}, \dot{s}=0$ ), as well as the output/capital ratios $f(\gamma k, \delta u h) / k$ and $g((1-\gamma) k,(1-\delta) u h) / k$. Equivalently, $f\left(\gamma, \delta u \frac{h}{k}\right)$ and $g\left((1-\gamma),(1-\delta) u \frac{h}{k}\right)$ are constant in a BGP due to the assumed homogeneity of degree one of $f$ and $g$. Based on this, we derive from (82)-(87), along with (35)-(37), a system of 6 equations in the unknowns $(c / k),(h / k), l, u, s, \nu$ (namely, system (40)-(45)), that must necessarily be satisfied in a BGP solution.

From (82), taking logs and differentiating both sides with respect to time, we obtain:

$$
\frac{\dot{\pi}_{1}}{\pi_{1}}=-\left[1-a_{1}(1-\varepsilon)\right] \nu
$$

which, in combination with (86) and the homogeneity of degree zero of $f_{K}, f_{L}, g_{K}, g_{L}$, yields (40). Equation (41) is trivially obtained by imposing the BGP conditions to (36). From (83), taking logs and differentiating both sides with respect to time, we again obtain:

$$
\frac{\dot{\pi}_{2}}{\pi_{2}}=-\left[1-a_{1}(1-\varepsilon)\right] \nu\left(=\frac{\dot{\pi}_{1}}{\pi_{1}}\right)
$$

and using (41):

$$
\frac{\dot{\pi}_{2}}{\pi_{2}}=a_{1}(1-\varepsilon) \nu+\theta-\psi(1-l-s-u) .
$$

Combining with (87) one finally obtains (42). Equation (43) is trivially obtained by imposing the BGP conditions to (35). In order to derive (44), we start from (84). By multiplying both sides of (84) by $h l s / c$, one obtains:

$$
\delta l \frac{s h}{c} \pi_{1} f_{L}\left(\gamma, \delta u \frac{h}{k}\right)+(1-\delta) l \frac{s h}{c} \pi_{3} g_{L}\left[(1-\gamma),(1-\delta) u \frac{h}{k}\right]=\psi l \frac{s h}{c} \pi_{2} .
$$

Note that combining (82) and (85) yields:

$$
\frac{a_{3}}{a_{1}} c \pi_{1}=\psi s h \pi_{2}+s h \pi_{3},
$$

whereas combination of $(82)$ and (83) results in:

$$
\psi s h \pi_{2}=\frac{a_{2}}{a_{1}} s \pi_{1} \frac{c}{l} .
$$

Therefore, (89) can be rewritten using (90), as follows:

$$
\frac{a_{3}}{a_{1}} c \pi_{1}=\frac{a_{2}}{a_{1}} s \pi_{1} \frac{c}{l}+s h \pi_{3},
$$

from which:

$$
\frac{s h}{c} \pi_{3}=\frac{a_{3}}{a_{1}} \pi_{1}-\frac{a_{2}}{a_{1}} s \pi_{1} \frac{1}{l} .
$$

By replacing (91) on the left-hand side of (88) and (90) on the right-hand side, one finally gets (44). Finally, (45) is trivially obtained by dividing by $k$ both sides of (37).

In order to obtain alternative forms (47), (48) for conditions (40), (44), respectively, we start from Euler's theorem for constant returns to scale homogeneous function $F$, namely

$$
F\left(x_{1}, x_{2}, \ldots, x_{I}\right)=\sum_{i=1}^{I} x_{i} F_{i}\left(x_{1}, x_{2}, \ldots, x_{I}\right),
$$


by which (70) can be rewritten as (we omit arguments):

$$
g=(1-\gamma) g_{K}+(1-\delta) u \frac{h}{k} g_{L}
$$

By combining (92) with equilibrium condition (45) one gets:

$$
(1-\delta) g_{L}=\frac{s}{u} \xi
$$

where $\xi=\xi\left(u, \frac{h}{k}\right):=1-(1-\gamma) g_{K} / g$. Substitution into (44) yields:

$$
\frac{a_{2}}{a_{1}}=\frac{\xi}{u}\left(\frac{a_{3}}{a_{1}} l-\frac{a_{2}}{a_{1}} s\right)+l \delta f_{L} \frac{h}{k} \frac{k}{c},
$$

from which (48) is obtained. The latter equation also shows that:

$$
\frac{a_{2}}{a_{1}} \frac{1}{l} \frac{c}{k}-\delta f_{L} \frac{h}{k}=\frac{\xi}{u} \frac{1}{l} \frac{c}{k}\left(\frac{a_{3}}{a_{1}} l-\frac{a_{2}}{a_{1}} s\right) .
$$

Substitution of (93) into (40) finally yields (47).

We now provide a few details on why BGP equations (40)-(45) turn out to be greatly simplified in the case of the PE (Section 3.3). From assuming $a_{3} \rightarrow 0, s \rightarrow 0$ and $\gamma, \delta \rightarrow 1$, it is clear that (45) becomes an identity and conditions (41), (42) and (43) are easily reduced to their counterparts (60), (61) and (62), respectively. Starting from condition (48), equivalent to (44) for $s \neq 0$, one easily obtains (63). Likewise, although condition (40) does not immediately suggest (59) as its counterpart in the $\mathrm{PE}$, the equivalent condition $(47)$ does the job. ${ }^{21}$

\subsection{Proof of Proposition 1}

From (41), $\theta=\psi\left(1-l^{A}-s^{A}-u^{A}\right)-\nu^{A}=\psi\left(1-l^{B}-s^{B}-u^{B}\right)-\nu^{B}$ and therefore

$$
\nu^{B}-\nu^{A}=\psi\left(\lambda^{B}-\lambda^{A}\right), \quad \psi>0,
$$

where $\lambda=1-l-s-u$. This proves $(50)$.

Similarly, by specializing (42) to different BGPs one obtains

$$
\rho-n=\psi\left(u^{A}+s^{A}-\frac{a_{3}}{a_{2}} l^{A}\right)+a_{1}(1-\varepsilon) \nu^{A}=\psi\left(u^{B}+s^{B}-\frac{a_{3}}{a_{2}} l^{B}\right)+a_{1}(1-\varepsilon) \nu^{B},
$$

or equivalently

$$
a_{1}(1-\varepsilon)\left(\nu^{B}-\nu^{A}\right)=\psi\left[\left(u^{A}-u^{B}\right)+\left(s^{A}-s^{B}\right)+\frac{a_{3}}{a_{2}}\left(l^{B}-l^{A}\right)\right] .
$$

Note that from $u=1-l-s-\lambda$ and from (94) it follows that

$$
u^{A}-u^{B}=\left(l^{B}-l^{A}\right)+\left(s^{B}-s^{A}\right)+\left(\lambda^{B}-\lambda^{A}\right)=\left(l^{B}-l^{A}\right)+\left(s^{B}-s^{A}\right)+\frac{1}{\psi}\left(\nu^{B}-\nu^{A}\right) .
$$

One can thus rewrite the equation (95) as follows

$$
\nu^{B}-\nu^{A}=\frac{\psi}{\tau}\left(1+\frac{a_{3}}{a_{2}}\right)\left(l^{A}-l^{B}\right),
$$

\footnotetext{
${ }^{21}$ Note that in the case of most common production functions, quantities $r\left(u, \frac{h}{k}\right)$ and $\xi\left(u, \frac{h}{k}\right)$ admit finite limits as $\gamma, \delta \rightarrow 1$. See below on Section 4 for the case of Cobb-Douglas technology.
} 
where $\tau:=1-a_{1}(1-\varepsilon)>0$ for any $\varepsilon>0$. This proves (51).

Finally, from (96) one obtains

$$
\frac{a_{3}}{a_{2}}\left(l^{B}-l^{A}\right)=\frac{\tau}{\psi} \frac{a_{3}}{a_{2}+a_{3}}\left(\nu^{A}-\nu^{B}\right) .
$$

Replacing into (95) yields:

$$
\left(1-\frac{\tau a_{2}}{a_{2}+a_{3}}\right)\left(\nu^{B}-\nu^{A}\right)=\psi\left[\left(u^{A}+s^{A}\right)-\left(u^{B}+s^{B}\right)\right] .
$$

Since:

$$
1-\frac{\tau a_{2}}{a_{2}+a_{3}}=\frac{a_{3}+a_{1} a_{2}(1-\varepsilon)}{a_{2}+a_{3}},
$$

part (52)-(54) of the statement follows.

Appendix 6.7 provides a further set of relations which involve, in particular, the levels of variables $(c / k)$ and $(h / k)$ at different BGPs. They are derived under constant returns to scale Cobb-Douglas technology. Unlike implications (50)-(54), such additional conditions are no longer independent of the exogenously assumed intersectoral capital shares and turn out to be too cumbersome to be usefully interpreted in economic terms. However they may provide the basis for further investigation. Moreover, in Appendix 6.7 the comparison across BGPs will be specialized to the PE, thus providing an alternative and more direct proof of the comparative results derived by Ladrón-de Guevara et al. [1999].

\subsection{Proof of Proposition 2}

Solving for $\theta$ from both (41) and (60) and equating one gets:

$$
\psi \lambda^{P E}-\nu^{P E}=\psi \lambda^{S E}-\nu^{S E}
$$

i.e.

$$
\nu^{S E}-\nu^{P E}=\psi\left(\lambda^{S E}-\lambda^{P E}\right)
$$

where $\lambda^{P E}:=1-l^{P E}-u^{P E}, \lambda^{S E}:=1-l^{S E}-u^{S E}-s$. This proves (64) and incidentally shows that the growth rate differential is proportional to the differential of time spent on human capital formation.

Combining (42) and (61) one obtains (under the assumption $a_{1}=a$ )

$$
\psi u^{P E}+a_{1}(1-\varepsilon) \nu^{P E}=\psi\left(u^{S E}+s\right)+a_{1}(1-\varepsilon) \nu^{S E}-\frac{a_{3}}{a_{2}} \psi l^{S E},
$$

which implies, given $a_{3}>0$ :

$$
\psi\left(u^{S E}+s\right)+a_{1}(1-\varepsilon) \nu^{S E}>\psi u^{P E}+a_{1}(1-\varepsilon) \nu^{P E},
$$

or equivalently:

$$
a_{1}(1-\varepsilon)\left(\nu^{S E}-\nu^{P E}\right)>\psi\left[u^{P E}-\left(u^{S E}+s\right)\right],
$$

which proves $(65)$ for $0<\varepsilon<1$. For completeness, note that $u^{P E}<\left(u^{S E}+s\right)$ for $\varepsilon=1$ (independent of the growth differential), whereas for $\varepsilon>1$ one obtains that $u^{S E}+s \leq u^{P E} \Longrightarrow \nu^{S E}<\nu^{P E}$.

Remember that $u^{P E}=1-l^{P E}-\lambda^{P E}$ and $u^{S E}+s=1-l^{S E}-\lambda^{S E}$. We can rewrite (98) as:

$$
a_{1}(1-\varepsilon)\left(\nu^{S E}-\nu^{P E}\right)>\psi\left[\left(l^{S E}-l^{P E}\right)+\left(\lambda^{S E}-\lambda^{P E}\right)\right] .
$$

By combining (99) and (97) we can write:

$$
\tau\left(\nu^{S E}-\nu^{P E}\right)<\psi\left(l^{P E}-l^{S E}\right),
$$

where $\tau:=1-a_{1}(1-\varepsilon)>0$ for any $\varepsilon>0$. This proves (66). 


\subsection{BGP conditions with Cobb-Douglas technology}

As is well known, for the Cobb-Douglas function (with constant returns to scale)

$$
F\left(x_{1}, x_{2}, \ldots, x_{I}\right):=A \prod_{i=1}^{I} x_{i}^{b_{i}}, \quad \sum_{i=1}^{I} b_{i}=1,
$$

marginal products admit the following representation in terms of total output $F$ :

$$
F_{i}\left(x_{1}, x_{2}, \ldots, x_{I}\right)=\frac{b_{i}}{x_{i}} F\left(x_{1}, x_{2}, \ldots, x_{I}\right)
$$

In particular, given production functions (70) and (70), quantity $\frac{h}{k} \delta f_{L}\left(\gamma, \delta u \frac{h}{k}\right)$ in equations (40) and (44) becomes:

$$
\frac{h}{k} \delta f_{L}\left(\gamma, \delta u \frac{h}{k}\right)=\frac{1-\beta}{u} f\left(\gamma, \delta u \frac{h}{k}\right),
$$

whereas quantities (46) and (49) take the simplified forms:

$$
r\left(u, \frac{h}{k}\right)=\frac{\eta}{1-\eta} u \frac{h}{k}, \quad \xi\left(u, \frac{h}{k}\right)=1-\eta
$$

respectively. The above simplifications, in addition to the change of variables $\Phi:=f\left(\gamma, \delta u \frac{h}{k}\right)=$ $B \gamma^{\beta}\left(\delta u \frac{h}{k}\right)^{1-\beta}$ from which:

$$
\frac{h}{k}=\frac{1}{\delta u}\left(\frac{\Phi}{B \gamma^{\beta}}\right)^{\frac{1}{1-\beta}},
$$

finally result in conditions (71)-(73) for the case of Cobb-Douglas technology.

\subsection{More on the comparison of BGPs in the SE and the PE}

Consider two BGPs in the SE. With the usual notation, it follows immediately from (72) that:

$$
\nu^{B}-\nu^{A}=\Phi^{B}-\Phi^{A}-\left[\left(\frac{c}{k}\right)^{B}-\left(\frac{c}{k}\right)^{A}\right],
$$

by which the growth differential turns out to be given by the output-capital ratio differential net of the differential between consumption-capital ratios. However, this simple linear relation between BGP quantities hides a far more complicated relation between $\Phi^{B}, \Phi^{A}$ and $(c / k)^{B},(c / k)^{A}$. As a matter of fact, from (76) we may write:

$$
\frac{a_{2}}{a_{1}} \frac{1}{l} \frac{c}{k}=\frac{1-\eta}{u+(1-\eta) s} \frac{a_{3}}{a_{1}} \frac{c}{k}+\frac{(1-\beta) \Phi}{u+(1-\eta) s}
$$

and therefore

$$
\frac{a_{2}}{a_{1}} \frac{1}{l} \frac{c}{k}-\frac{(1-\beta) \Phi}{u}=\frac{1-\eta}{u+(1-\eta) s}\left[\frac{a_{3}}{a_{1}} \frac{c}{k}-\frac{s}{u}(1-\beta) \Phi\right] .
$$

By replacing the latter quantity in the right-hand side of (71), one obtains a further equivalent formulation of condition (71):

$$
\rho+\sigma+\tau \nu=\Omega(u, s) \Phi+\omega(u, s) \frac{a_{3}}{a_{1}} \frac{c}{k},
$$


where

$$
\omega(u, s):=\frac{\eta u}{u+(1-\eta) s}, \quad \Omega(u, s):=\beta+(1-\beta) \frac{\eta s}{u+(1-\eta) s}=\beta+(1-\beta) \frac{s}{u} \omega(u, s) .
$$

Therefore, it follows from (101) that (we omit arguments for quantities $\omega$ and $\Omega$ ):

$$
\tau\left(\nu^{B}-\nu^{A}\right)=\Omega^{B} \Phi^{B}-\Omega^{A} \Phi^{A}+\frac{a_{3}}{a_{1}}\left[\omega^{B}\left(\frac{c}{k}\right)^{B}-\omega^{A}\left(\frac{c}{k}\right)^{A}\right] .
$$

Substitution of (100) into (103) finally yields

$$
\left(\tau-\Omega^{B}\right) \Phi^{B}-\left(\tau-\Omega^{A}\right) \Phi^{A}=\left(\tau+\frac{a_{3}}{a_{1}} \omega^{B}\right)\left(\frac{c}{k}\right)^{B}-\left(\tau+\frac{a_{3}}{a_{1}} \omega^{A}\right)\left(\frac{c}{k}\right)^{A},
$$

which makes clear that $\Phi^{B}-\Phi^{A}$ and $(c / k)^{B}-(c / k)^{A}$ are not connected in a linear fashion across different BGPs. ${ }^{22}$

From (100), (103) and (104) it is possible to derive a set of inequalities which would formally complement those included in Proposition 1. However, they would be too complicated to be discussed and interpreted satisfactorily in the general case of the SE. Moreover, this enlarged set of conditions requires that all parameters of the model are kept fixed when comparing two BGPs. This means that it is only valid for the comparison between multiple coexisting BGPs of the same economy. As already discussed in Section 3.2, Proposition 1 is valid under a broader range of situation including, in particular, the comparison between the Balanced Growth dynamics of the same economy before and after an exogenous perturbation of the parameters reflecting the intersectoral allocation of physical and human capital.

The remainder of this Appendix specializes the above discussed conditions to the case of the PE, thus providing a full set of relations between growth rate differentials and differentials of other variables in two BGPs. ${ }^{23}$ Note first that conditions (50)-(51) are still valid for the PE:

$$
\nu^{B} \gtreqless \nu^{A} \Longleftrightarrow \lambda^{B} \gtreqless \lambda^{A} \Longleftrightarrow l^{B} \lesseqgtr l^{A}
$$

whereas conditions (52)-(54) reduce to:

$$
\begin{gathered}
\text { for } 0<\varepsilon<1, \nu^{B} \gtreqless \nu^{A} \Longleftrightarrow u^{B} \lesseqgtr u^{A} ; \\
\quad \text { for } \varepsilon=1, u^{B}=u^{A} ; \\
\text { for } \varepsilon>1, \nu^{B} \gtreqless \nu^{A} \Longleftrightarrow u^{B} \gtreqless u^{A} .
\end{gathered}
$$

Note that, in the PE, quantities defined in (102) reduce trivially to $\omega(u, s)=\eta, \Omega(u, s)=\beta$. Therefore, (104) and (103) are simplified, respectively, to

$$
\begin{aligned}
(\tau-\beta)\left(\Phi^{B}-\Phi^{A}\right) & =\tau\left[\left(\frac{c}{k}\right)^{B}-\left(\frac{c}{k}\right)^{A}\right], \\
\Phi^{B}-\Phi^{A} & =\frac{\tau}{\beta}\left(\nu^{B}-\nu^{A}\right) .
\end{aligned}
$$

\footnotetext{
${ }^{22}$ However, they turn out to be connected in a linear fashion in the case of the PE. See below on this Section and Ladrón-de Guevara et al. [1999].

${ }^{23}$ Based on the above discussion, this set of relations among BGPs must be intended to be valid for the case of multiple BGPs for a fixed parameter setting.
} 
From the last two equations is follows that (for $\tau \neq 0$ )

$$
\left(\frac{c}{k}\right)^{B}-\left(\frac{c}{k}\right)^{A}=\left(\frac{\tau}{\beta}-1\right)\left(\nu^{B}-\nu^{A}\right) .
$$

Unlike in the SE, equations (105), (106) and (107) demonstrate that $\Phi^{B}-\Phi^{A},(c / k)^{B}-(c / k)^{A}$ and $\nu^{B}-\nu^{A}$ are connected in a simple, proportional fashion in the PE. In particular, the differential in output per capita, $\Phi^{B}-\Phi^{A}$, is positively proportional to $\nu^{B}-\nu^{A}$, whereas the differential in consumption to capital ratio, $(c / k)^{B}-(c / k)^{A}$, is positively or negatively proportional to $\nu^{B}-\nu^{A}$ depending on the sign of quantity $\tau / \beta-1$. Therefore, as $\tau:=1-\alpha(1-\varepsilon)$, the relation between $(c / k)^{B}-(c / k)^{A}$ and $\nu^{B}-\nu^{A}$ may be summarized as follows:

$$
\begin{gathered}
\text { for } 0<\varepsilon<1-\frac{1-\beta}{a}, \nu^{B} \gtreqless \nu^{A} \Longleftrightarrow\left(\frac{c}{k}\right)^{B} \lesseqgtr\left(\frac{c}{k}\right)^{A} ; \\
\text { for } \varepsilon=1-\frac{1-\beta}{a},\left(\frac{c}{k}\right)^{B}=\left(\frac{c}{k}\right)^{A} ; \\
\text { for } \varepsilon>1-\frac{1-\beta}{a}, \nu^{B} \gtreqless \nu^{A} \Longleftrightarrow\left(\frac{c}{k}\right)^{B} \gtreqless\left(\frac{c}{k}\right)^{A},
\end{gathered}
$$

where only the third case is possible if $a+\beta \leq 1$.

Now consider condition (100) specialized to the PE, where in particular:

$$
\Phi^{B}:=f\left(1, u^{B}\left(\frac{h}{k}\right)^{B}\right), \Phi^{A}:=f\left(1, u^{A}\left(\frac{h}{k}\right)^{A}\right) .
$$

One can show that, for $0<\varepsilon \leq 1$, the BGP characterized by larger growth is also the less physicalcapital intensive (and thus the more human-capital intensive). This was previously proven in the case of the PE by Ladrón-de Guevara et al. [1999], through comparative statics arguments. Instead, the proof provided in this Appendix is based on the simple inequalities developed above and on the change of variable performed in Section 4 , by which $(h / k)$ is conveniently replaced by variable $\Phi$ in the analysis of the BGPs. Note that condition (63) for the PE reads, under Cobb-Douglas technology:

$$
\frac{1-a}{a}=\frac{l}{u}(1-\beta) \Phi \frac{k}{c}
$$

Therefore, for a pair of coexisting BGPs it follows that:

$$
\frac{l^{B}}{u^{B}} \Phi^{B}\left(\frac{k}{c}\right)^{B}=\frac{l^{A}}{u^{A}} \Phi^{A}\left(\frac{k}{c}\right)^{A} .
$$

Assume now $\nu^{B}>\nu^{A}$ and remember that this also implies $\Phi^{B}>\Phi^{A}, l^{B}<l^{A}$ and $u^{B} \lesseqgtr u^{A}$ (depending on whether $\varepsilon \lesseqgtr 1$ ). Consider the case $0<\varepsilon \leq 1$. In this case it is easily seen from the above conditions that $\nu^{B}>\nu^{A}$ (and thus $\Phi^{B}>\Phi^{A}$ ) implies $u^{B} \leq u^{A}$. This fact, combined with (108) and the monotonicity of $\Phi$ with respect to its second argument, necessarily implies that $(h / k)^{B}>(h / k)^{A}$. Therefore we conclude that:

$$
\text { for } 0<\varepsilon \leq 1, \quad \nu^{B}>\nu^{A} \Longrightarrow\left(\frac{k}{h}\right)^{B}<\left(\frac{k}{h}\right)^{A} \text {. }
$$




\section{References}

J. Alonso-Carrera and X. Raurich. Growth, sectoral composition, and the evolution of income levels. Journal of Economic Dynamics and Control, 34(12):2440-2460, 2010.

C. Azariadis, B. L. Chen, C. H. Lu, and Y. C. Wang. A two-sector model of endogenous growth with leisure externalities. Journal of Economic Theory, 2013.

G. S. Becker. A theory of the allocation of time. The Economic Journal, 75(299):493-517, 1965.

J. Benhabib and R. Perli. Uniqueness and indeterminacy: on the dynamics of endogenous growth. Journal of Economic Theory, 63(1):113-142, 1994.

E. W. Bond, P. Wang, and C. K. Yip. A general two-sector model of endogenous growth with human and physical capital: balanced growth and transitional dynamics. Journal of Economic Theory, 68 (1):149-173, 1996 .

D. Cass. Optimum growth in an aggregative model of capital accumulation. The Review of Economic Studies, 32(3):233-240, 1965.

A. Chanda and C. Dalgaard. Dual economies and international total factor productivity differences: Channelling the impact from institutions, trade, and geography. Economica, 75(300):629-661, 2008.

A. C. Chu. Global poverty reduction and pareto-improving redistribution. Macroeconomic Dynamics, 16(04):605-624, 2012.

J. C. Córdoba and M. Ripoll. Agriculture and aggregation. Economics Letters, 105(1):110-112, 2009.

C. Echevarria. Changes in sectoral composition associated with economic growth. International Economic Review, pages 431-452, 1997.

M. B. El-hadj. A three-sector model of structural transformation and economic development. In $D E-$ GIT Conference Papers, number c014_039. DEGIT, Dynamics, Economic Growth, and International Trade, 2009.

T. C. Koopmans. On the concept of optimal economic growth. Cowles Foundation Discussion Papers, 1963.

A. Ladrón-de Guevara, S. Ortigueira, and M. S. Santos. A two-sector model of endogenous growth with leisure. The Review of Economic Studies, 66(3):609-631, 1999.

J. Laitner. Structural change and economic growth. The Review of Economic Studies, 67(3):545-561, 2000.

R. E. Lucas. On the mechanics of economic development. Journal of Monetary Economics, 22(1): $3-42,1988$.

N. G. Mankiw, D. Romer, and D. N. Weil. A contribution to the empirics of economic growth. The quarterly Journal of Economics, 107(2):407-437, 1992.

K. Mino. Indeterminacy in two-sector models of endogenous growth with leisure. Unpublished, 2002.

C. B. Mulligan and X. Sala-i Martin. Transitional dynamics in two-sector models of endogenous growth. The Quarterly Journal of Economics, 108(3):739-773, 1993. 
L. R. Ngai and C. A. Pissarides. Structural change in a multisector model of growth. The American Economic Review, 97(1):429-443, September 2007.

S. Ortigueira. A dynamic analysis of an endogenous growth model with leisure. Economic Theory, 16 (1):43-62, 2000.

I. N. Psarianos. A note on work-leisure choice, human capital accumulation, and endogenous growth. Research in economics, 61(4):208-217, 2007.

F. P. Ramsey. A mathematical theory of saving. The Economic Journal, 38(152):543-559, 1928.

R. Rogerson. Structural transformation and the deterioration of european labor market outcomes. Technical report, National Bureau of Economic Research, 2007.

T. W. Schultz. Investment in human capital. The American Economic Review, pages 1-17, 1961.

R. M. Solow. A contribution to the theory of economic growth. The Quarterly Journal of Economics, 70(1):65-94, 1956.

I. Steedman. Consumption takes time: implications for economic theory. Routledge, 2003.

H. Uzawa. Optimum technical change in an aggregative model of economic growth. International Economic Review, 6(1):18-31, 1965.

K. Wong and C. K. Yip. Education, economic growth, and brain drain. Journal of Economic Dynamics and Control, 23(5):699-726, 1999. 


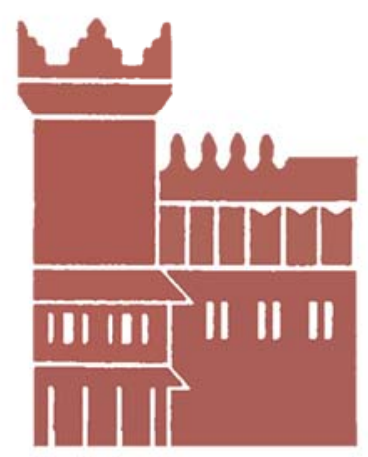

Alma Mater Studiorum - Università di Bologna DEPARTMENT OF ECONOMICS

Strada Maggiore 45

40125 Bologna - Italy

Tel. +39051 2092604

Fax +390512092664

http://www.dse.unibo.it 